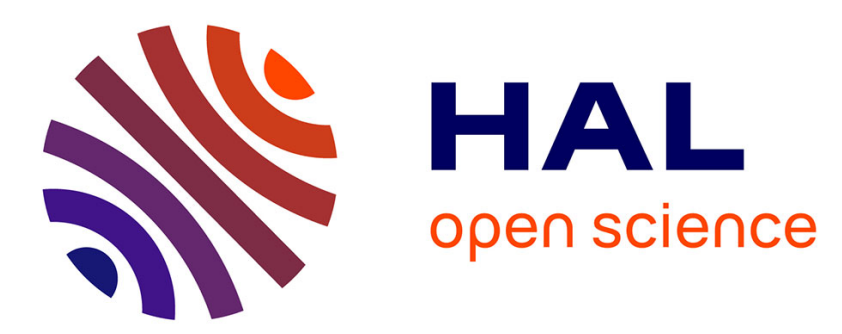

\title{
Directional Enlacement Histograms for the Description of Complex Spatial Configurations between Objects
}

Michaël Clément, Adrien Poulenard, Camille Kurtz, Laurent Wendling

\section{To cite this version:}

Michaël Clément, Adrien Poulenard, Camille Kurtz, Laurent Wendling. Directional Enlacement Histograms for the Description of Complex Spatial Configurations between Objects. IEEE Transactions on Pattern Analysis and Machine Intelligence, 2017, 10.1109/TPAMI.2016.2645151 . hal-01496903

\section{HAL Id: hal-01496903 https://hal.science/hal-01496903}

Submitted on 12 Jun 2017

HAL is a multi-disciplinary open access archive for the deposit and dissemination of scientific research documents, whether they are published or not. The documents may come from teaching and research institutions in France or abroad, or from public or private research centers.
L'archive ouverte pluridisciplinaire HAL, est destinée au dépôt et à la diffusion de documents scientifiques de niveau recherche, publiés ou non, émanant des établissements d'enseignement et de recherche français ou étrangers, des laboratoires publics ou privés. 


\title{
Directional Enlacement Histograms for the Description of Complex Spatial Configurations between Objects
}

\author{
Michaël Clément, Adrien Poulenard, Camille Kurtz, and Laurent Wendling
}

\begin{abstract}
The analysis of spatial relations between objects in digital images plays a crucial role in various application domains related to pattern recognition and computer vision. Classical models for the evaluation of such relations are usually sufficient for the handling of simple objects, but can lead to ambiguous results in more complex situations. In this article, we investigate the modeling of spatial configurations where the objects can be imbricated in each other. We formalize this notion with the term enlacement, from which we also derive the term interlacement, denoting a mutual enlacement of two objects. Our main contribution is the proposition of new relative position descriptors designed to capture the enlacement and interlacement between two-dimensional objects. These descriptors take the form of circular histograms allowing to characterize spatial configurations with directional granularity, and they highlight useful invariance properties for typical image understanding applications. We also show how these descriptors can be used to evaluate different complex spatial relations, such as the surrounding of objects. Experimental results obtained in the different application domains of medical imaging, document image analysis and remote sensing, confirm the genericity of this approach.
\end{abstract}

Index Terms—enlacement histograms, interlacement histograms, relative position descriptors, spatial relations, image understanding

\section{INTRODUCTION}

$\mathrm{T}$ HE spatial organization of objects plays a fundamental role in the perception of similarity between scenes or situations. Over the last decades, a large number of studies were conducted on spatial relations, in different scientific domains ranging from psychology, linguistics and human cognition [1], to artificial intelligence, pattern recognition and computer vision [2]. In particular, a strong focus has been placed on the proposition and the development of models for the evaluation of spatial relationships between objects present in image contents.

In this image understanding context, spatial relations are commonly divided into three main categories [3]: topological relations (e.g., are the objects overlapping?), distance relations (e.g., are the objects far from each other?) and directional relations (e.g., is an object to the left of another?). Even in simple situations, classical binary relations cannot satisfactorily model the spatial organization of objects. As potential solutions, several fuzzy models, designed for the interpretation of images [4], have been proposed in order to quantitatively capture the inherent imprecision carried by spatial relations [5], [6].

However, describing the relative position of objects arranged in complex spatial configurations remains a challenging task. As an illustrative example, let us consider the configurations presented in Fig. 1. Most existing models fall short at properly capturing the surrounding situation of (a), the imbrication of the objects of (b), or the more complex spatial interlacement of the spirals depicted in (c). In this article, we address the issue of modeling such complex spatial relationships, where the objects present some form of enlacement or interlacement. Even though the semantics of

The authors are with Université Paris Descartes, LIPADE, SIP team, 75006 Paris, France. Contact: michael.clement@parisdescartes.fr such relations remain difficult to define properly, it is worth mentioning that they only arise when the objects are concave, or composed of multiple connected components [7]. Consequently, they can be considered at the boundary between the directional and topological categories.

The underlying idea of this work is to be able to study and answer various questions regarding such complex relations, for instance:

- To which degree is an object enlaced by another object? To which degree are two objects interlaced together? Given an interlacement configuration, can we find a dominant orientation?

- How to distinguish different interlaced configurations of objects? Given two situations, which one is the most interlaced?

The rest of this article is organized as follows. Section 2 reviews some related work on the modeling of spatial relations in images. In Section 3, some useful definitions and notations are established, and we more formally state the problem studied in this research work. Then, our approach for the quantitative description of complex spatial configurations is presented in Section 4: we introduce the enlacement and interlacement histograms, which are relative position descriptors relying on directional information between the objects. We also put an emphasis on their invariance properties and their algorithmic implementation. In Section 5, we further explore how evaluations of different complex spatial relations can be derived from our proposed descriptors. Section 6 gathers experimental validations conducted on images from different application domains. Finally, conclusions and perspectives will be found in Section 7. 


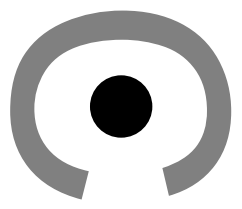

(a)

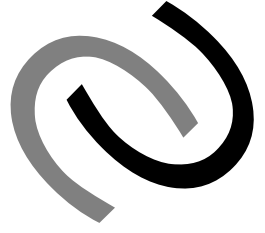

(b)

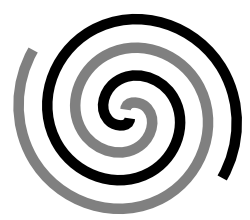

(c)
Fig. 1. The goal of this article is to study the following questions: is $A$ enlaced by $B$ ? Is $B$ enlaced by $A$ ? Are the two objects interlaced? (black: object $A$, gray: object $B$ )

\section{Related Work}

It has been highlighted in [8] that a distinction should be made between a spatial relationship and the relative position of an object with regards to another. There is indeed a strong duality between the two concepts.

On the one hand, a fuzzy evaluation of a spatial relation between two objects, such as "to the left of", allows to describe their relative position. For instance, the fuzzy landscape framework [9] is oriented towards this type of evaluations. This approach is based on a fuzzy modeling of given spatial relations directly in the image space, using morphological operations. Typical applications include for example graph-based face recognition [10], brain segmentation from MRI [11], or handwritten text recognition [12].

On the other hand, the relative position of an object with regards to another one can have a representation of its own, from which it is then possible to derive evaluations of spatial relations. Such relative position descriptors include angle histograms [13] or force histograms [14]. Force histograms have proven to be quite powerful relative position descriptors. They have a known behavior with regards to affine transformations [15], and efficient algorithms have been designed for their computation [16]. Force histograms are used in several application domains such as linguistic descriptions [17], scene matching [18] or content-based image retrieval [19], [20]. More recently, a new relative position descriptor has been proposed in [21], allowing to capture different spatial relations based on Allen temporal intervals [22]. For a more detailed review of existing relative position descriptors, the interested reader can refer to [23]. It can also be noted that spatial information can also be captured at a finer scale within a local features framework, for example inspired by the works of [24], [25].

Although both types of approaches (fuzzy evaluations and relative position descriptors) allow to assess elementary directional relations in a quantitative way, they cannot straightforwardly be extended to deal with more specific spatial relations. For instance, in Fig. 1 (a), a typical evaluation of the "surrounding" spatial relation would imply the combination of different elementary directional relations: an object $A$ has to be simultaneously in all directions of another object $B$ in order to be considered surrounded by it. This approach is intuitive, but often leads to ambiguous results in practice, because the surrounding object $B$ is also simultaneously considered in all directions of the surrounded object $A$. The same kind of observation can be made for the more complex spatial configurations of (b) and (c). Consequently, there is an important need for models dedicated to the proper handling of specific and contextual spatial relations, and several works were proposed in this direction.

A classical complex spatial relation is the "surrounding". The notion of visual surroundedness was first introduced in [26]. This idea has been further investigated in [27] with a dedicated fuzzy landscape approach. The surrounding relation has also been studied in [28] using force histograms, but the approach suffers from the ambiguities mentioned previously. Another specific spatial relation is "between". It has been thoroughly studied in [7] using definitions based on convex hulls, and morphological operations. In particular, applications of this spatial relation in digital histopathology have been proposed in [29]. The authors of [30] proposed an approach to measure the spatial relation "along". In the field of remote sensing imaging, methods were proposed for the detection of the alignment and parallelism of geographical objects [31], as well as the "to go across" relation [32].

To the best of our knowledge, even though different specific spatial relations were studied in the literature, no approach has been proposed to tackle the issue of measuring complex situations of enlacement and interlacement, such as the ones presented in Fig. 1.

\section{Definitions and Problem Statement}

In this section, we introduce some useful notations and definitions, in particular regarding the handling of objects. We also describe the intuitive idea behind what is intended with the terms enlacement and interlacement. Finally, we formally state the problem studied in this article, specifying some requirements around which we designed our approach.

\subsection{Two-Dimensional Objects}

In the Euclidean space, a two-dimensional object $A$ can be defined as a nonempty, bounded set of points, i.e., $A \subset \mathbb{R}^{2}$. Equivalently, an object $A$ can be defined by its characteristic (membership) function $\mathbb{1}_{A}: \mathbb{R}^{2} \rightarrow\{0,1\}$, which associates a binary value to each point $(x, y) \in \mathbb{R}^{2}$. This definition of an object can easily be extended to the fuzzy case, and an object $A$ is then defined as a fuzzy subset represented by its membership function $\mu_{A}: \mathbb{R}^{2} \rightarrow[0,1]$. More generally, it is possible to interpret two-dimensional objects as functions of compact support from $\mathbb{R}^{2}$ to $\mathbb{R}$, allowing for a more analytical manipulation. Therefore, for the sake of genericity, an object $A$ will be identified in the following by its representative function $f_{A}: \mathbb{R}^{2} \rightarrow \mathbb{R}$.

\subsection{Longitudinal Cuts}

For ease of notation, throughout the rest of the paper, the Euclidean plane $\mathbb{R}^{2}$ and the complex plane $\mathbb{C}$ are equivalently identified by the isomorphism $(x, y) \in \mathbb{R}^{2} \mapsto(x+i y) \in \mathbb{C}$.

Let $\theta \in \mathbb{R}$ be an orientation angle, and $\rho \in \mathbb{R}$ a distance from the origin. We define the oriented line of angle $\theta$ at the altitude $\rho$ by the following non-finite set:

$$
\Delta^{(\theta, \rho)}=\left\{e^{i \theta}(t+i \rho), t \in \mathbb{R}\right\},
$$

where $t \in \mathbb{R}$ denotes the coordinate of a point relatively to the line $\Delta^{(\theta, \rho)}$. Let $\Delta^{(\theta, \rho)}$ be such an oriented line, and $A$ an object. The subset $A \cap \Delta^{(\theta, \rho)}$ represents a one-dimensional 


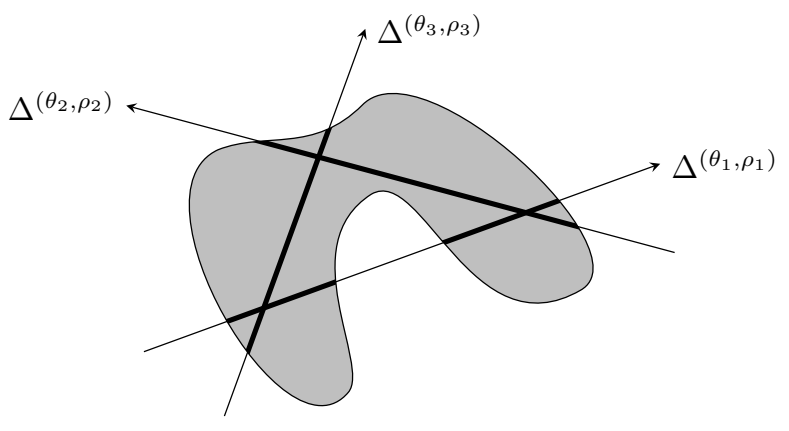

Fig. 2. Different longitudinal cuts of a binary object. An oriented line $\Delta^{(\theta, \rho)}$ slices the object into either an empty, or a finite set of segments.

slice of the object $A$, also called a longitudinal cut. In the case of binary objects, such a longitudinal cut of $A$ is either empty (the oriented line does not cross the object) or composed of a finite number of segments, as illustrated in Fig. 2. In the continuous case, similarly to the functional notation of objects defined previously, a longitudinal cut of $A$ along the line $\Delta^{(\theta, \rho)}$ can be interpreted as a function $f_{A}^{(\theta, \rho)}$ defined as:

$$
\begin{aligned}
f_{A}^{(\theta, \rho)}: \mathbb{R} & \longrightarrow \mathbb{R} \\
t & \longmapsto f_{A}\left(e^{i \theta}(t+i \rho)\right),
\end{aligned}
$$

where $t$ represents a point on the one-dimensional line $\Delta^{(\theta, \rho)}$ and $f_{A}^{(\theta, \rho)}(t)$ gives us the representative value of this point on the plane, according to the object $A$.

\subsection{Enlacement and Interlacement}

As stated previously, the goal of this article is to study complex spatial relationships such as how an object is enlaced by another, or how two objects are interlaced. Even though such spatial relationships are easily apprehended by human perception, they are usually vaguely defined and particularly ambiguous, making them difficult to evaluate quantitatively. Therefore, we propose to fix some vocabulary terms to designate these semantic concepts, and we explain what is intended behind these terms.

The term enlacement characterizes and generalizes the idea that an object $A$ (commonly called the argument) is somehow between, surrounded or squeezed by another object $B$ (the referent). Depending on the configuration, the relation " $A$ is enlaced by $B$ " may or may not hold, to a certain degree. It is a relative spatial relationship, in the sense that it is always defined on an object with regards to another. Also note that the relation is not symmetric: $A$ can be enlaced by $B$, but the opposite might not hold.

Subsequently, the term interlacement is defined as a mutual enlacement of two objects. The term encompasses the idea that the two objects mutually surround each other (but not necessarily to the same extent). It is not a relative definition, but an absolute measurement based on the spatial configuration of the two objects.

These definitions can be intuitively illustrated by the examples of Fig. 1. In (a), the relation " $A$ is enlaced by $B$ " holds to a certain degree, but not the opposite, so the relation " $A$ and $B$ are interlaced" does not hold. This is a typical surrounding situation. In (b), the enlacement between the objects is now mutual: there are parts of $A$ surrounded by parts of $B$, and conversely. Therefore, according to our definition, we can consider that the two objects are interlaced, to some extent. In (c), the objects are strongly interlaced, because they mutually surround each other, in all directions.

\subsection{Problem Statement}

We seek to satisfactorily describe the enlacement and the interlacement between any given couple of two-dimensional objects, be it in the binary, fuzzy, or real case, while precisely taking into account the shape of the objects. The objects can also be composed of multiple connected components. Such a description should be able to discriminate different configurations of enlacement and interlacement between couples of objects, and should allow for both local and global evaluations to be derived.

Therefore, the problem may be apprehended in a similar fashion to a classical pattern recognition task, inducing for example invariance constraints with regards to rotations, translations and scaling transformations. Our goal is to build a quantitative relative position descriptor which is intuitive, fast to compute and applicable to any objects. Such requirements are useful in many image analysis tasks, and constitute the basis on which our approach is built.

\section{Directional Enlacement of Objects}

In this section, we present the theoretical framework developed to quantitatively describe the enlacement between two-dimensional objects. As stated in the previous section, this problem is complex, especially because we have to take into account the shape of the objects. The reasoning process applied to build our approach is inspired by the one employed in [13], [14] for the representation of directional spatial relations. It is mainly based on the assumption that a global evaluation of directional spatial relations can be achieved by aggregating local spatial information between individual points belonging to the objects.

\subsection{One-Dimensional Enlacement}

Let $(A, B)$ be a couple of two-dimensional objects. Our goal is to describe the enlacement of $A$ with regards to $B$. In order to measure such a relationship, the idea is to use specific relative positions of individual points $a_{i} \in A$ and $b_{i} \in B$ as arguments to put in favor of the proposition " $A$ is enlaced by $B^{\prime \prime}$. In the case of measuring the enlacement, we have to capture the occurrences of points of $A$ being somehow between points of $B$. However, it is difficult to precisely identify such occurrences in the two-dimensional plane. Therefore, we propose to treat points of the objects in a more convenient one-dimensional case, where the notion of betweenness can be precisely defined without ambiguities.

Let us first restrict ourselves to objects defined in the binary case, and let us consider an oriented line $\Delta^{(\theta, \rho)}$. As explained previously, such a line goes through two finite sets of points of $A$ and $B$, where each point can be uniquely defined by its one-dimensional coordinate relative to $\Delta^{(\theta, \rho)}$. In this situation, an elementary occurrence of $A$ being enlaced by $B$ can be defined as an ordered triplet of points 


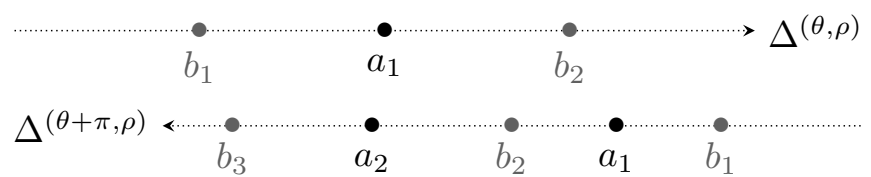

Fig. 3. Illustration of the enlacement between points along a line for two configurations. Along the oriented line $\Delta^{(\theta, \rho)}$, the ordered triplet $\left(b_{1}, a_{1}, b_{2}\right)$ is the only argument to be put in favor of the proposition " $A$ is enlaced by $B$ ". Along the opposite oriented line $\Delta^{(\theta+\pi, \rho)}$, the arguments in favor of the proposition can be represented by the set of ordered triplets $\left\{\left(b_{1}, a_{1}, b_{2}\right),\left(b_{1}, a_{1}, b_{3}\right),\left(b_{1}, a_{2}, b_{3}\right),\left(b_{2}, a_{2}, b_{3}\right)\right\}$.

$\left(b_{i}, a_{k}, b_{j}\right) \in B \times A \times B$ where $b_{i}<a_{k}<b_{j}$. This idea is illustrated in Fig. 3 . In the first configuration, the only argument that can be put in favor of " $A$ is enlaced by $B$ " is the ordered triplet $\left(b_{1}, a_{1}, b_{2}\right)$. Also, note that there is no argument in favor of the enlacement of $B$ by $A$. In the second configuration, which is oriented in the opposite direction, several occurrences of primitive enlacement situations can be put in favor of " $A$ is enlaced by $B$ ". In fact, the set of triplets $\left\{\left(b_{1}, a_{1}, b_{2}\right),\left(b_{1}, a_{1}, b_{3}\right),\left(b_{1}, a_{2}, b_{3}\right),\left(b_{2}, a_{2}, b_{3}\right)\right\}$ completely represents the arguments in favor of the situation. Also note that for the inverse relation, only $\left(a_{1}, b_{2}, a_{2}\right)$ can be put in favor of " $B$ is enlaced by $A$ ".

Therefore, to globally measure the enlacement of $A$ with regards to $B$ along a one-dimensional oriented line $\Delta^{(\theta, \rho)}$, we count the number of occurrences of such situations among points of the two objects located on such a line. In other words, we want to capture the overall quantity of $A$ located simultaneously after and before parts of $B$. Let us recall that the functions $f_{A}^{(\theta, \rho)}$ and $f_{B}^{(\theta, \rho)}$ designate longitudinal cuts of our objects along the oriented line $\Delta^{(\theta, \rho)}$. Based on this notation, the quantity of $A$ located after a point $x$ on a line $\Delta^{(\theta, \rho)}$ is given by:

$$
\int_{x}^{+\infty} f_{A}^{(\theta, \rho)}(y) \mathrm{d} y
$$

Geometrically speaking, if the object $A$ is defined in the binary case, this integral represents the cumulated length of the segments on the interval $[x,+\infty[$ of the considered longitudinal cut. Consequently, to capture the quantity of $A$ located after points of $B$ on this line, we have:

$$
\int_{-\infty}^{+\infty} f_{B}^{(\theta, \rho)}(x) \int_{x}^{+\infty} f_{A}^{(\theta, \rho)}(y) \mathrm{d} y \mathrm{~d} x .
$$

Symmetrically, the quantity of $A$ located before parts of $B$ is obtained with:

$$
\int_{-\infty}^{+\infty} f_{A}^{(\theta, \rho)}(x) \int_{x}^{+\infty} f_{B}^{(\theta, \rho)}(y) \mathrm{d} y \mathrm{~d} x .
$$

The goal is then to combine these two quantities, in order to take into account both parts of $B$ before and after parts of $A$, leading us to the following general definition of the one-dimensional enlacement.

Definition 1. Let $f$ and $g$ be two bounded measurable functions with compact support from $\mathbb{R}$ to $\mathbb{R}$. The enlacement of $f$ with regards to $g$ is defined as:

$$
E(f, g)=\int_{-\infty}^{+\infty} g(x) \int_{x}^{+\infty} f(y) \int_{y}^{+\infty} g(z) \mathrm{d} z \mathrm{~d} y \mathrm{~d} x .
$$

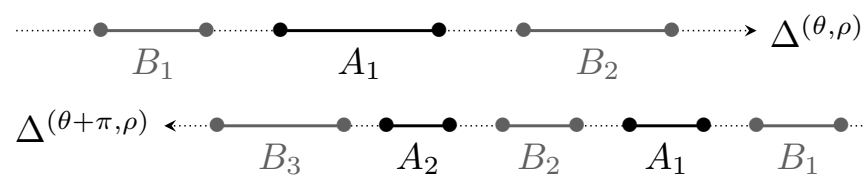

Fig. 4. Illustration of the enlacement between segments along a line for two configurations ( $A_{i}$ and $B_{i}$ denote the lengths of the segments). On the first line, the enlacement of $A$ segments by $B$ segments in direction $\theta$ is given by $B_{1} A_{1} B_{2}$. On the second line, the enlacement of $A$ segments by $B$ segments in direction $\theta+\pi$ is $\left(B_{1} A_{1} B_{2}\right)+\left(B_{1} A_{1} B_{3}\right)+$ $\left(B_{1} A_{2} B_{3}\right)+\left(B_{2} A_{2} B_{3}\right)$.

This generic definition between two real-valued functions can be applied to longitudinal cuts $f_{A}^{(\theta, \rho)}$ and $f_{B}^{(\theta, \rho)}$. The value $E\left(f_{A}^{(\theta, \rho)}, f_{B}^{(\theta, \rho)}\right)$ represents the overall enlacement of $A$ by $B$ along the oriented line $\Delta^{(\theta, \rho)}$. For example, if the objects are defined in the binary case, it corresponds to the total number of ordered triplets of points on the oriented line, which can be seen as arguments to put in favor of the proposition " $A$ is enlaced by $B$ " in the direction $\theta$.

As an illustration of this definition, Fig. 4 extends the configurations of Fig. 3 from points to segments $\left(A_{i}\right.$ and $B_{i}$ denote the segment lengths). The enlacement of $A$ segments by $B$ segments along the first line $\Delta^{(\theta, \rho)}$ is given by $B_{1} A_{1} B_{2}$. Along the second line $\Delta^{\theta+\pi, \rho}$, the total enlacement of $A$ segments by $B$ segments is $\left(B_{1} A_{1} B_{2}\right)+\left(B_{1} A_{1} B_{3}\right)+$ $\left(B_{1} A_{2} B_{3}\right)+\left(B_{2} A_{2} B_{3}\right)$. Symmetrically, the enlacement of $B$ segments by $A$ segments is represented by $A_{1} B_{2} A_{2}$.

\subsection{Enlacement of Objects}

In the previous section, we introduced the notion of enlacement between real-valued functions, which can be applied to the representative functions of objects sliced along a one-dimensional oriented line. In the following, we explain how this definition can be further extended to handle twodimensional objects as a whole.

Let $\theta \in \mathbb{R}$ be a fixed orientation angle. The set of all parallel lines $\left\{\Delta^{(\theta, \rho)}, \rho \in \mathbb{R}\right\}$ in this direction will slice any object into a set of longitudinal cut functions. To measure the enlacement of an object with regards to another in this direction, we aggregate the one-dimensional enlacement values obtained for each of these longitudinal cuts, as expressed by the following definition.

Definition 2. Let $\theta \in \mathbb{R}$ be an orientation angle, and let $A$ and $B$ be two objects. The enlacement of $A$ by $B$ in the direction $\theta$ is defined by:

$$
E_{A B}(\theta)=\int_{-\infty}^{+\infty} E\left(f_{A}^{(\theta, \rho)}, f_{B}^{(\theta, \rho)}\right) \mathrm{d} \rho .
$$

The enlacement of $A$ by $B$ in the direction $\theta$ is defined as the integral of the one-dimensional enlacement over all parallel oriented lines of direction $\theta$. The principle of this definition is illustrated in Fig. 5. In the binary case, this definition corresponds to a number of triplets of points to put in favor of " $A$ is enlaced by $B$ " along the longitudinal cuts of the objects going in this direction. Intuitively, it can be interpreted as the quantity of $B$ traversed while sliding the object $A$ in the direction $\theta$, with regards to the quantity of $B$ located on the opposite direction. 


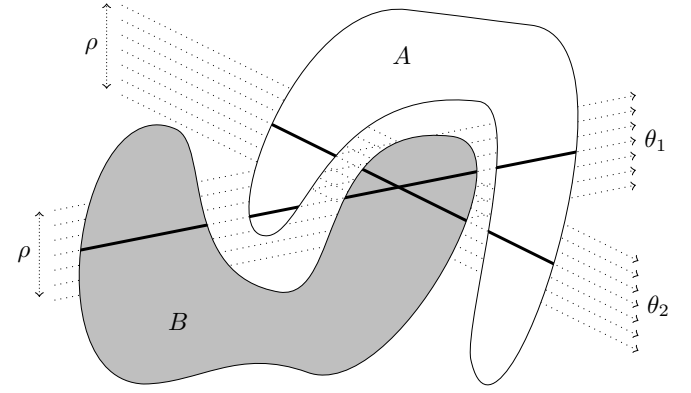

Fig. 5. The set of all parallel lines going in a given direction slices the objects $A$ and $B$ into sets of longitudinal cuts. The combination of the one-dimensional enlacement values obtained for each of these longitudinal cuts allows to measure the overall enlacement in this direction.

The scalar value resulting from this definition is therefore essentially directional, and depends on the spatial configuration between the two objects. It is based on the mass distribution of the objects in space, according to the set of parallel lines of orientation $\theta$. For example, if no points of $A$ are captured by points of $B$ in the direction $\theta$, then we will have $E_{A B}(\theta)=0$. On the contrary, if large parts of $A$ are trapped by large parts of $B$ when looking at the objects in the direction $\theta$, then $E_{A B}(\theta)$ will increase.

In order to illustrate this definition, Fig. 6 shows the enlacement profiles obtained along the set of directions $\theta \in[0,2 \pi]$ for a series of toy examples. The objects are composed of multiple connected components, properly displaying the behavior of the two possible enlacement profiles ( $E_{A B}$ and $E_{B A}$ ) with regards to increasingly complex configurations. For the sake of simplicity, the enlacement here is essentially horizontal: the profiles are equal to 0 in most directions, with two isolated peaks centered around directions $\theta=0$ and $\theta=\pi$. The most elementary situation of enlacement is the one depicted in (a), where only $A$ is enlaced by $B$ (and not the opposite, i.e., $E_{B A}(\theta)=0$ for any $\theta)$. In (b), the enlacement of the two objects is now mutual: one component of $B$ is trapped between two components of $A$, and conversely. The two enlacement profiles $E_{A B}$ and $E_{B A}$ are thus identical. In (c), one component of $B$ is trapped between three components of $A$, resulting in a $E_{B A}$ profile greater than $E_{A B}$. Finally, the large $E_{A B}$ peak in (d) is due to the three components of $A$ being completely trapped by the components of $B$ at the extremities. Note that these two components do not count towards $E_{B A}$, and therefore $E_{B A}$ is identical to the previous situation, because the same component of $B$ is still trapped by the components of $A$. These examples highlight the fact that the combination of both enlacement profiles $E_{A B}$ and $E_{B A}$ enables to precisely describe and discriminate the different configurations between a couple of objects $(A, B)$. This leads to the notion of the interlacement between objects, which will be further investigated in the next section.

In the following, we enumerate some of the mathematical properties of Definition 2 (proofs of these properties can be found in Appendix).

Property 1 (Periodicity). For any two objects $A$ and $B$, the directional enlacement $E_{A B}$ is periodic with period $\pi$, that is:

$$
\forall \theta \in \mathbb{R}, E_{A B}(\theta)=E_{A B}(\theta+k \pi), k \in \mathbb{Z} .
$$

Property 2 (Translation). For any two objects $A$ and $B$, the directional enlacement $E_{A B}$ is invariant with regards to translations. If we denote by $T_{v}$ the translation by a vector $v \in \mathbb{C} \simeq \mathbb{R}^{2}$, we have:

$$
E_{T_{v}(A) T_{v}(B)}(\theta)=E_{A B}(\theta) .
$$

Property 3 (Rotation). For any two objects $A$ and $B$, the directional enlacement $E_{A B}$ is quasi-invariant with regards to rotations. Let $\alpha \in \mathbb{R}$ be a rotation angle and let $R_{\alpha}$ be the associated rotation transformation, then we have:

$$
E_{R_{\alpha}(A) R_{\alpha}(B)}(\theta)=E_{A B}(\theta-\alpha) .
$$

Property 4 (Scaling 1). For any two objects $A$ and $B$, the directional enlacement $E_{A B}$ is quasi-invariant with regards to scaling transformations. Let $\lambda \in \mathbb{R}$ be a scaling factor and let $S_{\lambda}$ be the associated scaling transformation, then we have:

$$
E_{S_{\lambda}(A) S_{\lambda}(B)}(\theta)=\lambda^{4} E_{A B}(\theta) .
$$

The $\pi$-periodicity of $E_{A B}(\theta)$ highlighted by Property 1 is interesting from an algorithmic point of view, because it allows to restrict the computation to the $[0, \pi]$ interval. The other properties guarantee that the directional enlacement is invariant with regards to translations, that rotations only result in circular shifts, and that scaling transformations are linear with regards to the scaling factor.

\subsection{Enlacement and Interlacement Histograms}

In the following, we present how to extend Definition 2 in order to build our enlacement and interlacement descriptors. To better reflect the overall enlacement of an object by another, the scalar enlacement value in a given direction should be relative to the overall area of the two objects considered. In such a way, the enlacement of different couples of objects having the same area (but different shapes) could be compared. Let us define the area $\|A\|_{1}$ of an object $A$ by:

$$
\|A\|_{1}=\iint_{\mathbb{R}^{2}}\left|f_{A}(x, y)\right| \mathrm{d} x \mathrm{~d} y .
$$

The directional enlacement descriptor is then given in the following definition.

Definition 3. Let $A$ and $B$ be two objects. The directional enlacement descriptor of $A$ with regards to $B$ is defined by the following function:

$$
\begin{aligned}
\mathcal{E}_{A B}: \mathbb{R} & \longrightarrow \mathbb{R} \\
\theta & \longmapsto \frac{E_{A B}(\theta)}{\|A\|_{1}\|B\|_{1}} .
\end{aligned}
$$

The enlacement descriptor $\mathcal{E}_{A B}$ takes the form of a function, which associates to each angle $\theta$ the enlacement value of $A$ by $B$ in this specific direction, normalized by the areas of the objects. By construction, each value of this function corresponds to an overall number of elementary occurrences of enlacement, obtained by considering longitudinal cuts of the objects in direction $\theta$. The descriptor can be interpreted as a circular histogram, which we call the $\mathcal{E}$-histogram.

The normalization allows to better represent the idea that the global enlacement is measured by aggregating local occurrences of enlacement between points along onedimensional lines. Also, it allows us to introduce the following scale invariance property (proof provided in Appendix). 

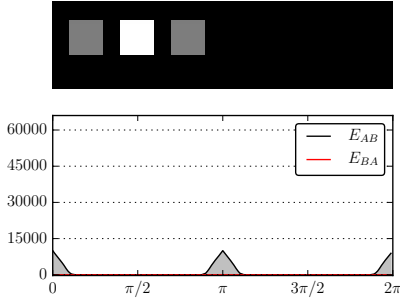

(a)
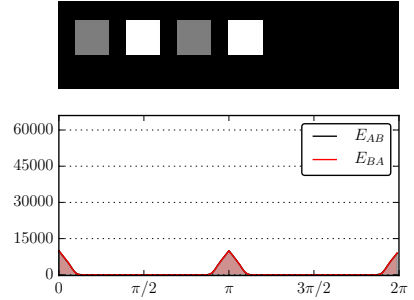

(b)
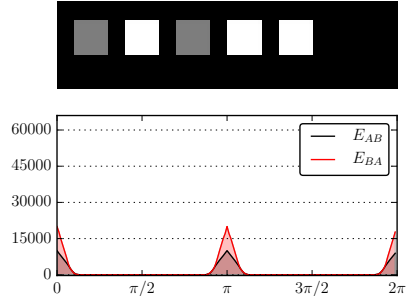

(c)
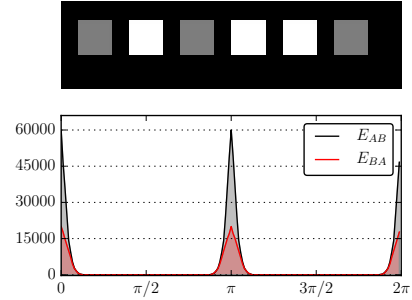

(d)

Fig. 6. Evolution of the directional enlacement profiles $E_{A B}$ and $E_{B A}$ on a series of simple examples where objects are composed of multiple connected components (white: object $A$, gray: object $B$ ), illustrating increasingly complex configurations in the horizontal directions. In these examples, each square has an area of $10 \times 10$ pixels (in (b), the two curves overlap).

Property 5 (Scaling 2). For any two objects $A$ and $B$, the enlacement histogram $\mathcal{E}_{A B}$ is invariant with regards to scaling transformations. Let $\lambda \in \mathbb{R}$ be a scaling factor and let $S_{\lambda}$ be the associated scaling transformation, then we have:

$$
\mathcal{E}_{S_{\lambda}(A) S_{\lambda}(B)}(\theta)=\mathcal{E}_{A B}(\theta) .
$$

It is easy to show that Properties 1,2 and 3 are preserved from $E_{A B}$ for the enlacement histogram $\mathcal{E}_{A B}$. With Property 5 deduced from Property 4 , we conclude that the $\mathcal{E}$-histogram between two objects is invariant with regards to translations and scaling transformations, and quasiinvariant to rotations (a rotation of angle $\alpha$ implies a shift of $\alpha$ in the descriptor).

The enlacement histogram $\mathcal{E}_{A B}$ allows to quantitatively characterize how an object $A$ is enlaced by another object $B$. It is therefore a relative position descriptor (it describes the position of an object with regards to another). As a result, the descriptor $\mathcal{E}_{A B}$ on its own does not completely describe the spatial configuration of the couple of objects $(A, B)$. As highlighted in Fig. 6, the other descriptor $\mathcal{E}_{B A}$ is complementary to $\mathcal{E}_{A B}$ and should also be considered to describe the configuration. In the end, a combination of these two descriptors provides a description of the interlacement of the two objects (defined as a mutual enlacement in Sec. 3.3), which leads us to the following definition.

Definition 4. Let $(A, B)$ be a couple of objects. We define the directional interlacement descriptor of $(A, B)$ as the harmonic mean between their respective couple of directional enlacement descriptors $\left(\mathcal{E}_{A B}, \mathcal{E}_{B A}\right)$ :

$$
\mathcal{I}_{A B}(\theta)=\frac{2 \mathcal{E}_{A B}(\theta) \mathcal{E}_{B A}(\theta)}{\mathcal{E}_{A B}(\theta)+\mathcal{E}_{B A}(\theta)}
$$

As for the enlacement descriptor, the interlacement descriptor can be interpreted as a circular histogram, called the $\mathcal{I}$-histogram. By construction, the invariance properties are preserved for the interlacement histogram.

\subsection{Algorithm and Complexity}

The directional enlacement and interlacement descriptors have been defined in the continuous case, using analytical notations to manipulate objects. In particular, it allowed us to generalize the notion of objects and to put an emphasis on invariance properties. Hereinafter, we propose algorithmic considerations to implement the descriptor of enlacement in a more practical discrete case, where the objects are represented by rasterized images of finite sizes.

Let us consider a square image composed of $N$ pixels where $A$ and $B$ are defined as crisp (binary) objects. For a given direction $\theta$, the computation of $\mathcal{E}_{A B}(\theta)$ first requires to generate the set of parallel lines $\left\{\Delta^{(\theta, \rho)}, \rho \in \mathbb{R}\right\}$. In practice, a single straight line $\Delta^{(\theta, \rho)}$ is rasterized using the classical Bresenham line-drawing algorithm. The subsequent parallel lines are obtained by shifting this initial line, ensuring that each pixel is counted once and only once.

On a single line, the number of points is in $\mathcal{O}(\sqrt{N})$, the worst case appearing in the diagonal of a square image. The triple integral of Definition 1 is derived by an appropriate distribution of segment lengths products. The segment lengths are computed in an isotropic manner, taking into account the orientation angle. This handling of segments on a single line induces a cubic complexity, resulting in a computation in $\mathcal{O}(N \sqrt{N})$. Following this, the number of parallel lines is bounded by $\sqrt{N}$. Therefore, the computation of the enlacement histogram $\mathcal{E}_{A B}$ along a set of $k$ discretized directions has a worst-case complexity of $\mathcal{O}\left(k N^{2}\right)$. Note that this worst-case complexity is never achieved in practice, because it would imply extremely distorted objects where pixels of each object alternate with each other in a checkerboard pattern.

The number of directions $k$ is usually fixed to a few hundreds (in this paper, we fixed $k=180$ according to a similar strategy adopted for F-Histograms [14]). Note that the $\pi$-periodicity of the descriptor allows to optimize the computation (only the $[0, \pi]$ interval has to be considered). Using this discretization strategy, the actual runtime is approximately 1-2 seconds for a $500 \times 500$ image with an i7 CPU and a naive Python implementation. Also note that the computation is highly parallelizable, either in a multithreaded or a distributed environment. Finally, the handling of fuzzy objects can be achieved by considering a finite set of $m$ membership values, and using either a classical simple or double sum scheme [14].

\section{Evaluation of Complex Spatial Relations}

In this section, we propose different approaches to use the enlacement descriptors for the interpretation of complex spatial relationships between objects. The goal is to illustrate that these descriptors can be considered in a more classical evaluation framework, while highlighting the discriminative power lying in directional information when dealing with the enlacement and the interlacement of objects. 

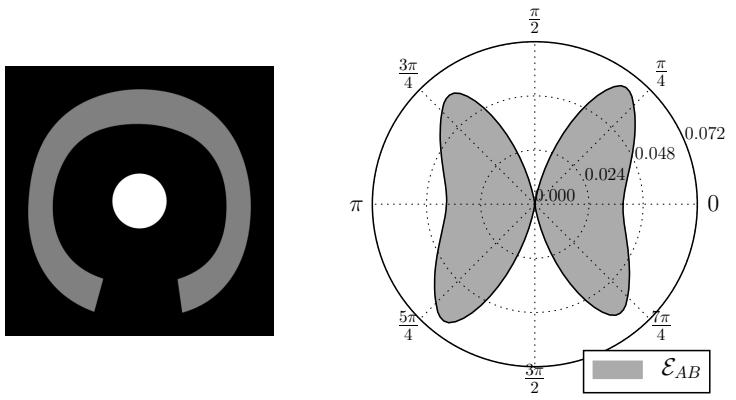

(a)

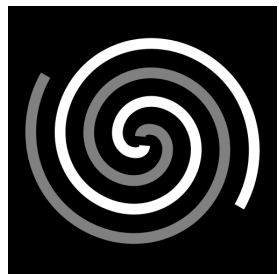

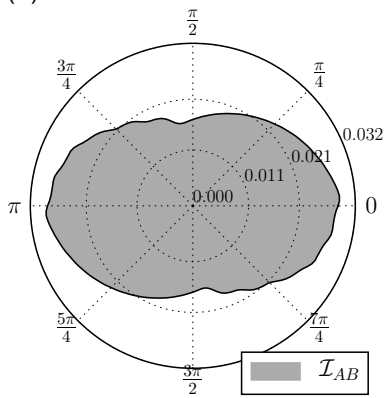

(c)
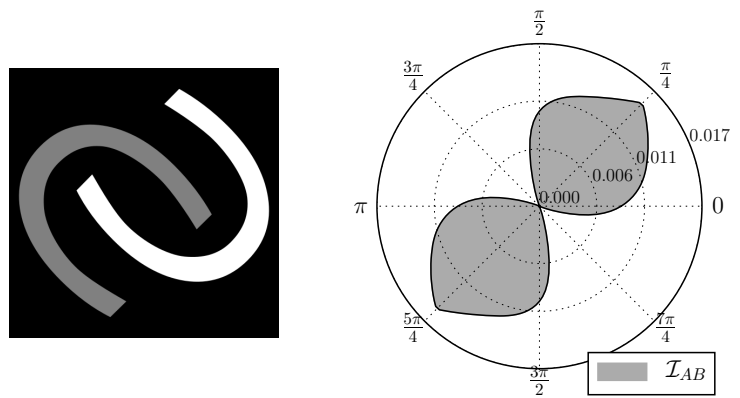

(b)

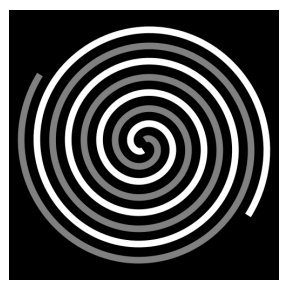

(d)

Fig. 7. Polar representations of the directional descriptors of (a) enlacement, and (b, c, d) interlacement obtained for the configurations introduced previously in Fig. 1 (white: object $A$, gray: object $B$ ).

\subsection{Enlacement and Interlacement}

The aim here is to evaluate the enlacement and the interlacement on different configurations of two objects using the descriptors proposed in the previous section. We propose to evaluate these spatial relations in directional manner, that is, we want to allow the evaluation of more specific spatial relations such as "enlaced/interlaced in direction $\theta$ ". Fig. 7 shows polar representations of the directional histograms obtained for the configurations introduced in Fig. 1. Notice that (a) shows the enlacement $\mathcal{E}_{A B}\left(\mathcal{E}_{B A}\right.$ is null), while (b), (c) and (d) present the interlacement $\mathcal{I}_{A B}$. This polar representation allows to effectively visualize the distribution of the enlacement/interlacement along the different directions. In the following, $\mathcal{H}_{A B}$ designates either the enlacement $\mathcal{E}_{A B}$ or interlacement $\mathcal{I}_{A B}$ (depending on the evaluation performed), normalized by its maximum value (it can then be assimilated to a fuzzy set).

A typical method to evaluate spatial relations is the fuzzy pattern matching approach [33]. The idea of this approach is to assimilate the directional histogram as a fuzzy set and to compare it to a fuzzy set modeling the desired spatial relation. The fuzzy set modeling the enlacement/interlacement in a specific direction is inspired by the one classically used to evaluate primitive directional relations (such as "to the right of"), for example in [13]. The fuzzy set centered in direction $\alpha$ is defined in [9] as $\mu_{\alpha}(\theta)=\mu_{0}(\theta-\alpha)$ where

$$
\mu_{0}(\theta)= \begin{cases}\cos ^{2}(\theta) & \text { if }-\frac{\pi}{2} \leq \theta \leq \frac{\pi}{2} \\ 0 & \text { otherwise. }\end{cases}
$$

In this work, this fuzzy set represents the degree of enlacement/interlacement in a specific direction $\alpha$. Subsequently, the directional evaluation of the spatial relation represented by $\mu_{\alpha}$ is obtained by both a necessity degree $N_{\alpha}$ and a possibility degree $\Pi_{\alpha}$, defined as follows:

$$
\begin{aligned}
\Pi_{\alpha}\left(\mathcal{H}_{A B}\right) & =\sup _{\theta \in[0,2 \pi]} \min \left(\mathcal{H}_{A B}(\theta), \mu_{\alpha}(\theta)\right), \\
N_{\alpha}\left(\mathcal{H}_{A B}\right) & =\inf _{\theta \in[0,2 \pi]} \max \left(1-\mathcal{H}_{A B}(\theta), \mu_{\alpha}(\theta)\right) .
\end{aligned}
$$

Note that the min and max operators could by replaced by any fuzzy $t$-norm and $t$-conorm according to the desired semantics. The necessity $N_{\alpha}$ represents a pessimist evaluation, while the possibility $\Pi_{\alpha}$ is an optimist evaluation. The interval $\left[N_{\alpha}, \Pi_{\alpha}\right]$ allows to visualize the confidence in the presence of a dominant orientation.

Tab. 1 shows the necessity-possibility intervals obtained for the configurations of Fig. 7. For the sake of clarity, we present the results obtained for four orientation angles only in the $[0, \pi]$ interval. This is due to the $\pi$-periodicity of the circular histograms. These results are consistent with the model: the possibility values allow to assess the confidence in the presence of enlacement/interlacement in this direction, while the necessity values only assess if the histogram matches the desired profile. For instance, in configuration (b), we obtain a necessity of 0.42 because the histogram matches relatively well with the profile in $\pi / 4$, but the necessity values are low for configurations (c) and (d) because the interlacement values are relatively high in most directions.

In our context, this approach does not allow for a proper evaluation of the overall quantity of enlacement or interlacement in a specific direction. As an alternative, we introduce the following averaging approach for the assessment of the enlacement and interlacement of objects. Let $\alpha \in[0,2 \pi]$ be a direction angle. The idea is to compute the area below $\mathcal{H}_{A B}$ on an interval of directions centered around $\alpha$. An 
TABLE 1

Evaluation of the enlacement and interlacement of objects in specific directions using a fuzzy pattern matching approach. (a), (b), (c) and (d) refer to the configurations of Fig. 7 .

\begin{tabular}{c|cccc} 
& 0 & $\frac{\pi}{4}$ & $\frac{\pi}{2}$ & $\frac{3 \pi}{4}$ \\
\hline (a) & {$[0.22,0.75]$} & {$[0.06,0.99]$} & {$[0.22,0.75]$} & {$[0.01,0.94]$} \\
(b) & {$[0.17,0.82]$} & {$[0.42,1.00]$} & {$[0.18,0.83]$} & {$[0.00,0.56]$} \\
(c) & {$[0.28,1.00]$} & {$[0.10,0.89]$} & {$[0.00,0.72]$} & {$[0.11,0.89]$} \\
(d) & {$[0.14,1.00]$} & {$[0.07,0.95]$} & {$[0.00,0.86]$} & {$[0.05,0.93]$}
\end{tabular}

evaluation on the interval $\left[\alpha-\frac{\omega}{2}, \alpha+\frac{\omega}{2}\right]$ is given by:

$$
\frac{1}{\omega} \int_{\alpha-\frac{\omega}{2}}^{\alpha+\frac{\omega}{2}} \mathcal{H}_{A B}(\theta) \mathrm{d} \theta .
$$

The value $\alpha$ represents the direction in which we want to evaluate the spatial relation, while $\omega$ controls the width of the interval, allowing to measure either a local or a more global directional information. For example, the interval $\left[0, \frac{\pi}{2}\right]$ allows to measure the interlacement located in the top-right direction, while an evaluation on the entire $[0,2 \pi]$ interval measures the overall quantity of enlacement/interlacement in the configuration.

Tab. 2 shows the evaluation results obtained for the configurations of Fig. 7. For each configuration, evaluations are performed on successive intervals centered on $\alpha_{i}=\left\{0, \frac{\pi}{4}, \frac{\pi}{2}, \frac{3 \pi}{4}, \pi\right\}$, each of width $\omega=\frac{\pi}{2}$, as well as a global evaluation on $[0,2 \pi]$. For configuration (a), the evaluation is performed on the enlacement histogram, and we can observe higher values in the horizontal directions, with an overall enlacement of 0.61 . The lower enlacement in the vertical directions is due to the small hole in the bottom of object B. A more specific method for such "surrounding" configurations is introduced in the next section. Configuration (b) clearly highlights that the two objects are more interlaced in the vertical direction. Finally, the spirals (c) and (d) both result in a strong overall interlacement $(0.78$ and 0.90 respectively), while also featuring a stronger value in the horizontal direction. This dominant orientation is mainly due to the external endings of the spirals, leading to a deeper level of interlacement between the objects in this direction.

\subsection{Surrounding}

In Section 3, we defined the enlacement as a generalization of the notion of surroundedness between objects of arbitrarily complex shapes. Therefore, given two objects (for example configurations presented in Fig. 8), we should be able to extract relevant information from the enlacement histogram $\mathcal{E}_{A B}$ in order to evaluate to which degree one object is surrounded by the other. The spatial relation "surrounded by" is intrinsically difficult to evaluate, mainly because it is located at the boundary between a directional and a topological spatial relation. In the following study, we will focus mainly on directional information. Intuitively, and according to our definition, an object should be considered surrounded by another object if it is "enlaced" by it in "all" directions. The "all" directions point of view has been adopted by other works such as in [28] using F-Histograms and in [27] with a fuzzy landscape framework.
TABLE 2

Evaluation of the enlacement and interlacement of objects in specific directions using our averaging approach. (a), (b), (c) and (d) refer to the configurations of Fig. 7.

\begin{tabular}{c|ccccc|c} 
& {$\left[-\frac{\pi}{4}, \frac{\pi}{4}\right]$} & {$\left[0, \frac{\pi}{2}\right]$} & {$\left[\frac{\pi}{4}, \frac{3 \pi}{4}\right]$} & {$\left[\frac{\pi}{2}, \pi\right]$} & {$\left[\frac{3 \pi}{4}, \frac{5 \pi}{4}\right]$} & {$[0,2 \pi]$} \\
\hline (a) & 0.71 & 0.62 & 0.50 & 0.59 & 0.71 & 0.61 \\
(b) & 0.16 & 0.46 & 0.81 & 0.51 & 0.16 & 0.48 \\
(c) & 0.90 & 0.80 & 0.66 & 0.76 & 0.90 & 0.78 \\
(d) & 0.96 & 0.92 & 0.85 & 0.89 & 0.96 & 0.90
\end{tabular}

An evaluation of the spatial relation "surrounded by" for two objects $A$ and $B$ is a real number $\mathcal{S}_{A B} \in[0,1]$. When $\mathcal{S}_{A B}=1$, the object $A$ can be considered as "totally surrounded" by the object $B$, whereas if $\mathcal{S}_{A B}=0, A$ is "not surrounded at all" by $B$. Values in between should denote a degree of validity of the proposition. We propose a method to evaluate $\mathcal{S}_{A B}$ from the enlacement descriptor $\mathcal{E}_{A B}$. The descriptor $\mathcal{E}_{A B}$ is normalized by its maximum value, and can subsequently be interpreted as a repartition of the overall enlacement along the set of directions $\theta \in[0,2 \pi]$. For $\alpha \in[0,1]$, we define a cut of $\mathcal{E}_{A B}$ as $\mathcal{E}_{A B}^{\alpha}(\theta)=\min \left\{\alpha, \mathcal{E}_{A B}(\theta)\right\}$.

The idea is to compute the overall area below $\mathcal{E}_{A B}^{\alpha}$, with $\alpha$ being interpreted as a tolerance threshold. Therefore, we define the degree to which " $A$ is surrounded by $B$ " with tolerance $\alpha$ by:

$$
\mathcal{S}_{A B}(\alpha)= \begin{cases}\frac{1}{2 \pi} \int_{0}^{2 \pi} 1-\delta\left(\mathcal{E}_{A B}(\theta)\right) \mathrm{d} \theta & \text { if } \alpha=0 \\ \frac{1}{2 \alpha \pi} \int_{0}^{2 \pi} \mathcal{E}_{A B}^{\alpha}(\theta) \mathrm{d} \theta & \text { otherwise, }\end{cases}
$$

where $\delta$ denotes the function taking the value 1 in 0 , and is 0 elsewhere. For a tolerance of $\alpha=0$, the object is considered to be surrounded as long as the enlacement is not equal to zero, and only the overall area where the enlacement is equal to zero lowers the surrounding value $\mathcal{S}_{A B}(0)$. Similarly, when $\left.\left.\alpha \in\right] 0,1\right]$, as long as $\mathcal{E}_{A B}(\theta) \geq \alpha$, the object is considered to be surrounded. In order to enrich this approach, we compute $\mathcal{S}_{A B}(\alpha)$ for multiple values of $\alpha \in[0,1]$. Studying the evolution of the surrounding values $\mathcal{S}_{A B}(\alpha)$ allows to interpret the situation from different points of views, from pessimistic to optimistic, and to better take into account the complexity of this spatial relation.

Fig. 9 shows a box plot of the $\mathcal{S}_{A B}(\alpha)$ values obtained for the configurations of Fig. 8. It allows to visualize the mean (red square) and median (red bar), as well as the dispersion of the surrounding values in $[0,1]$, and the rectangular box is delimited by the first and third quartiles.

Configurations (a) to (d) are similar, in the sense that the shape of the objects is uniform. In situation (a), the object is completely surrounded, and as expected, the surrounding value is 1 for all tolerance thresholds. In situation (b), the object is only partially surrounded, resulting in a lower surrounding value around 0.55 . The object is also partially surrounded in situation (c), where half of the surrounding circle has been cut out. We can also notice that the uniformity of situations (b) and (c) results in a relatively compact repartition of the evaluations. In situation (d), small parts were added preventing the object to leave without crossing 


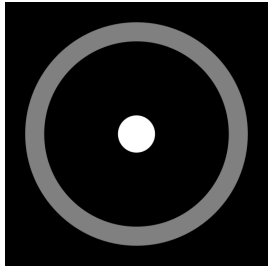

(a)

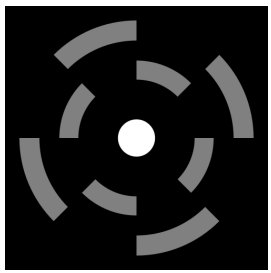

(d)

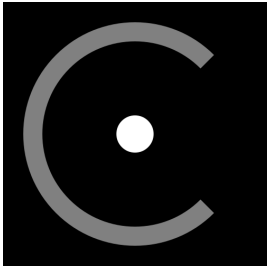

(b)

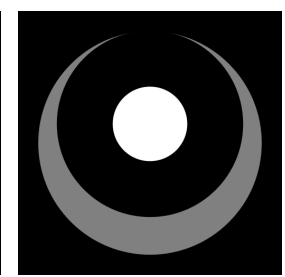

(e)

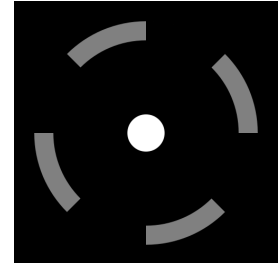

(c)

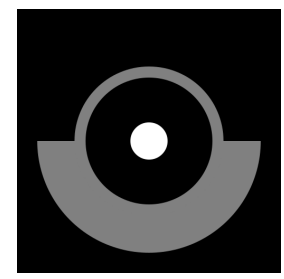

(f)
Fig. 8. Typical configurations where the degree of truth given to the proposition " $A$ is surrounded by $B$ " may vary (white: object $A$, gray: object $B)$.

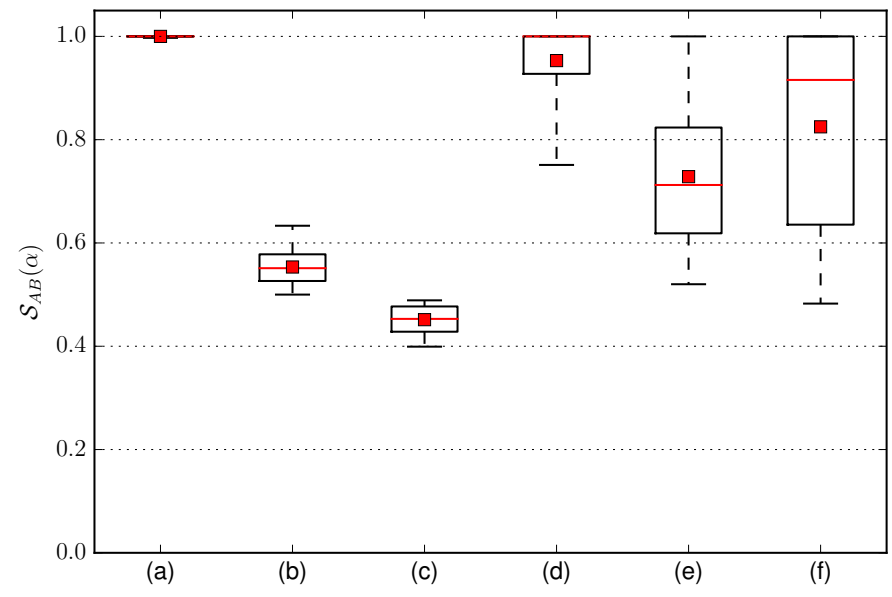

Fig. 9. Box plot of the surrounding values $\mathcal{S}_{A B}(\alpha)$ obtained for different tolerance values of $\alpha \in[0,1]$, for each configuration presented in Fig. 8 .

the surrounding object, and therefore most points of views are close to 1 , but with a wider spread of values.

Situations showed in (e) and (f) are different. For situation (e), the most optimist point of view gives a surrounding value of 1 , obtained for $\alpha=0$. It means that there exists no direction angle $\theta$ where the enlacement is equal to zero, or in other words, the object cannot leave without crossing at least small parts of the other object. However, because the top part of the surrounding object is thinner, the resulting surrounding values are relatively low on average, with a value around 0.5 for the most pessimist point of view. Finally, most values tend to agree that the object is surrounded in situation (f), and the optimist point of view is 1 . Yet, the object can leave by crossing smaller amounts of the surrounding object, and the pessimist point of view would say that the object is surrounded to an extent of 0.5 .

For comparison purposes, we also present in Tab. 3 the results provided by the main quantitative methods dedicated to the evaluation of the surrounding of objects: the approach of [28] using F-Histograms and the approach of
TABLE 3

Surrounding evaluations obtained for the approaches of [28] and [27], compared to the evaluations of our approach, for the different configurations of Fig. 8.

\begin{tabular}{c|c|c|c} 
& Matsakis et al. [28] & Vanegas et al. [27] & Our approach $\mathcal{S}_{A B}(\alpha)$ \\
\hline (a) & 0.99 & {$[1.00,1.00], 1.00$} & {$[1.00,1.00], 1.00 \pm 0.00$} \\
(b) & 0.50 & {$[0.70,0.79], 0.76$} & {$[0.50,0.63], 0.55 \pm 0.03$} \\
(c) & 0.74 & {$[0.50,0.54], 0.52$} & {$[0.40,0.49], 0.45 \pm 0.03$} \\
(d) & 0.94 & {$[0.93,1.00], 0.97$} & {$[0.75,1.00], 0.95 \pm 0.07$} \\
(e) & 0.16 & {$[0.76,0.90], 0.85$} & {$[0.52,1.00], 0.73 \pm 0.13$} \\
(f) & 0.25 & {$[0.94,1.00], 0.99$} & {$[0.48,1.00], 0.82 \pm 0.19$}
\end{tabular}

[27] with a fuzzy landscape framework. In [28], the authors aggregate different $\alpha$-cuts of the Force Histogram between the two objects, using a single sum scheme, which results in a single real-valued evaluation. The results of [27] are based on the definition of a specific fuzzy landscape, considering only the concavities of the surrounding object. We then obtain a necessity/possibility interval and an average value as the final evaluations. Here we did not apply the optional decision function as mentioned in [27], because it resulted in evaluations of 1.00 for most our proposed configurations. Even though the interpretation of the surrounding spatial relation is highly subjective, we believe that our approach allows for a richer interpretation of the configurations proposed in Fig. 8. Besides, the approach of [28] suffers from the "semantic inverse" paradox (i.e., the opposite surrounding evaluation of $B$ by $A$ gives the same results), while the approach of [27] is highly sensitive to discretization issues.

\section{EXPERIMENTAL VALIDATIONS}

In this section, we present different experimental applications designed to illustrate the genericity of our approach in various practical domains of image analysis and pattern recognition. We introduce applications in the contexts of medical imaging (characterization of blood vessels in retinal imaging pathologies), document image analysis (recognition of decorative drop caps) and remote sensing (classification of geographical urban objects).

\subsection{Overview of Comparative Methods}

We first introduce the different feature descriptors used as comparative methods in the following experiments, which can be considered as relevant baselines. The features used are Force Histograms [14] (FHistograms) and Generic Fourier Descriptors [34] (GFD). FHistograms are state-of-the-art relative position descriptors which focus on classic directional relations. In our experiments, FHistograms are computed with a constant force along a set of $k=180$ discrete directions, as for the interlacement descriptors. GFDs are wellknown binary shape descriptors capturing the invariant frequencies of shape patterns. We would like to emphasize that our goal is not to obtain the best reachable results for each considered application, but to illustrate that in practical tasks, our approach can lead to new insights in the characterization of complex spatial configurations. 

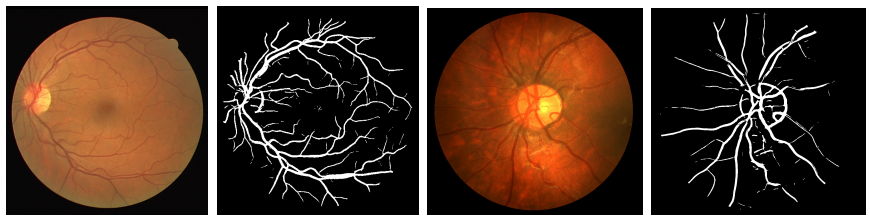

Fig. 10. Examples of retinal images from the DRIVE [35] and CHASEDB1 [36] datasets, with their respective blood vessels automatic segmentations provided by [37]. The first image corresponds to a retina from the DRIVE dataset (shifted optic disk), while the other comes from CHASEDB1 (centered optic disk).
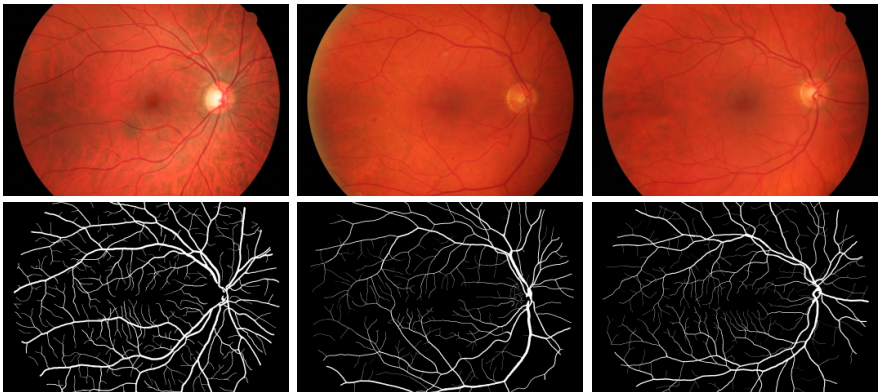

Fig. 11. Examples of retinal images from the HRF dataset [38], alongside their respective gold standard blood vessels segmentations. From left to right: healthy case, diabetic retinopathy, glaucoma.

\subsection{Blood Vessels Characterization in Retinal Imaging}

\subsubsection{Datasets}

In these experiments, we used three public datasets of fundus photographs of the retina: DRIVE [35], CHASEDB1 [36] and High-Resolution Fundus (HRF) [38]. These datasets are generally used to evaluate blood vessels segmentation methods, and are often provided with gold standard segmentations from ophthalmology experts. Here, we propose to use our descriptors to characterize the interlacement of the blood vessel structures within the rest of the fundus in retinal images. The DRIVE dataset is composed of 20 images where the optic disks are shifted from the center, while the CHASEDB1 dataset is composed of 20 images with centered optic disks. Fig. 10 presents some examples of these two specific datasets. The HRF dataset is composed of 45 images: 15 fundus from healthy patients, 15 with diabetic retinopathy symptoms and 15 with glaucoma symptoms. Fig. 11 presents some illustrative samples of this dataset.

\subsubsection{Experimental Protocol}

The first experimental application is designed to evaluate the robustness of our descriptors with regards to image segmentation issues. To this end, the goal was to perform a classification of the images from DRIVE and CHASEDB1, trying to discriminate vascular networks where the optic disk is shifted (DRIVE) from the ones where it is centered (CHASEDB1). The vascular networks were segmented from these images using an automatic blood vessel segmentation approach [37] achieving state-of-the-art results on these datasets. From these results, we then gradually added different types of random noise (salt \& pepper, Speckle and Gaussian noise) to the segmentations, which can be interpreted as increasingly strong segmentation errors.
TABLE 4

Comparative accuracy results for the noise robustness evaluation on the DRIVE and CHASEDB1 datasets.

\begin{tabular}{l|c|c|c} 
& GFD & FHistograms & Interlacement \\
\hline Speckle & $81.00 \pm 0.43$ & $96.50 \pm 0.03$ & $97.75 \pm 0.03$ \\
Gaussian & $93.75 \pm 0.47$ & $99.00 \pm 0.05$ & $99.25 \pm 0.03$ \\
Salt \& pepper & $94.25 \pm 0.10$ & $96.25 \pm 0.09$ & $98.75 \pm 0.02$
\end{tabular}

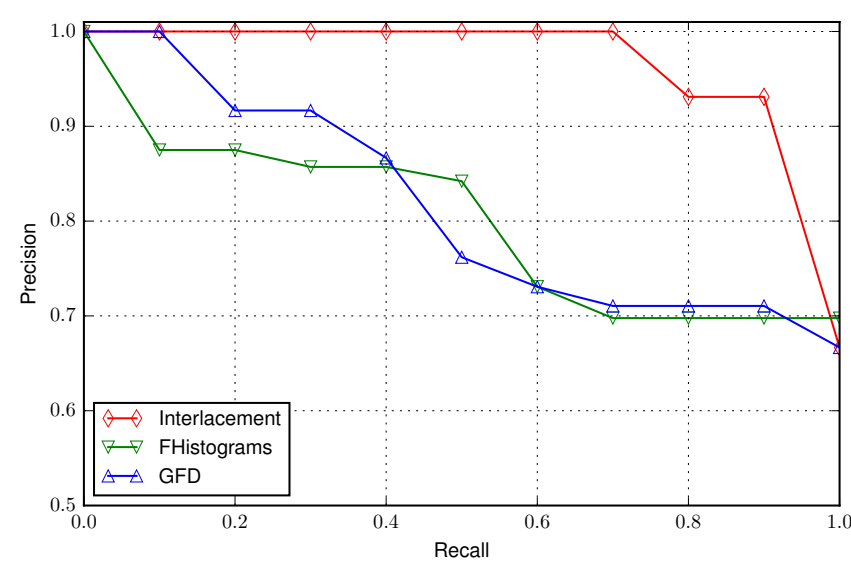

Fig. 12. Precision-recall curves obtained for the classification of retinal images from the HRF dataset, for interlacement descriptors $\mathcal{I}_{A B}$, FHistograms and GFDs.

The second application is the automatic classification of retinal pathologies. We used the images of the HRF dataset and their gold standard segmentations from ophthalmology experts. We split the images into two classes: healthy images and pathological images (including both diabetic retinopathy and glaucoma cases). Indeed, pathological blood vessels may be affected by artifacts (e.g., neovascularization, aneurysms, hemorrhages) and the spatial configurations of their networks may be impacted [39]. Note that other biological symptoms could be used to characterize these pathologies, but the objective here is to illustrate that interlacement descriptors can be used to model a part of the complexity of these objects.

For both applications, we compute interlacement descriptors $\mathcal{I}_{\mathcal{A B}}$ on segmented images, with $A$ being the blood vessels binary image, and $B$ being the rest of the fundus. FHistograms are computed on the same couple of objects, and GFDs on the blood vessels. Classification is achieved using the $k$-Nearest Neighbors algorithm (with $k=5$ ) with the proposed descriptors. We use a leave-one-out cross validation strategy (each test sample is compared to the rest of the dataset) and the $L_{2}$ norm to compare descriptors.

\subsubsection{Results}

Tab. 4 shows the results of the noise robustness experiment on the DRIVE and CHASEDB1 datasets, where we report mean classification accuracy for increasing amounts of noise added to the segmented images. Salt \& pepper was added with 10 increasing amounts in $[0,0.10]$, while Speckle and Gaussian were added with 10 increasing variances $\sigma \in[0,2]$. These results highlight the robustness 

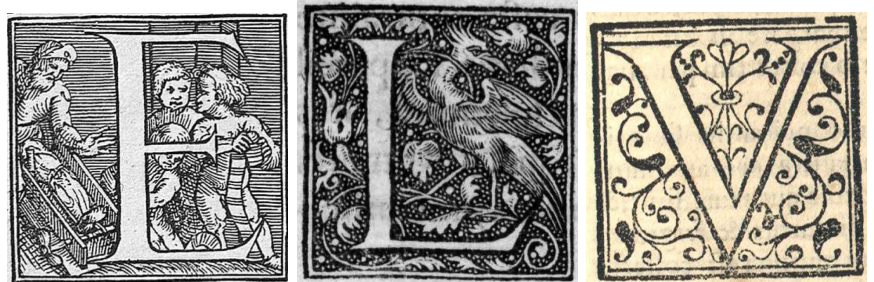

Fig. 13. Examples of images from the decorative drop caps dataset, with different background styles: hatchings, dotted, and mostly empty. These background styles present distinctive interlacement configurations.

of interlacement descriptors with regards to segmentation errors. They are significantly better than GFD descriptors at discriminating shifted and centered optic disks, and they slightly improve upon FHistograms, with lower standard deviations overall.

Fig. 12 shows the precision-recall curves obtained for the second proposed experiment: the classification of retinal pathologies. From these results, we can observe the capacity of interlacement descriptors to discriminate images issued from pathological patients from the healthy ones. They allow to efficiently characterize the artifact symptoms in the vascular networks for the diabetic and glaucoma cases, as opposed to the healthy ones.

\subsection{Recognition of Decorative Drop Caps}

\subsubsection{Dataset}

The dataset used for this application is a collection of decorative drop caps images extracted from ancient documents, in the context of the NaviDoMass project ${ }^{1}$. Drop caps recognition is a challenging task since these patterns can present various textured backgrounds, as well as complex ornamental decorations [40]. This task is of great interest for the digital preservation of historical document collections in libraries, museums and archives. In this work, we used a subset of 120 images classified by historians into three classes, each class representing a different background style (independently from the letter itself): hatchings, dotted and mostly empty, as illustrated in Fig. 13.

\subsubsection{Experimental Protocol}

The goal here is to classify drop caps images according to their background classes. The underlying insight for this application is that these different background styles may be characterized by distinctive interlacement patterns. First, the images are segmented into two layers, separating the ink pixels (foreground) from the paper ones (background). We used several well-known binary thresholding procedures in order to assess the performance stability of the approach with regards to potential segmentation errors. The thresholding methods used are Otsu (minimum intra-class variance), $L i$ (minimum cross-entropy), Isodata (inter-means) and Yen (maximum correlation with cost function). Then, for each image, the interlacement descriptor $\mathcal{I}_{A B}$ is computed between ink (object $A$ ) and paper (object $B$ ). The classification protocol is the same as for retinal images: $k-\mathrm{NN}$ classifier with $L_{2}$ norm, and leave-one-out cross validation.

1. http:/ /navidomass.univ-lr.fr/
TABLE 5

Comparative accuracy results for the classification of decorative drop caps with different segmentation methods.

\begin{tabular}{l|c|c|c} 
& GFD & FHistograms & Interlacement \\
\hline Otsu & 80.00 & 93.33 & 95.83 \\
Isodata & 79.17 & 95.00 & 95.83 \\
Li & 81.67 & 96.67 & 97.50 \\
Yen & 78.33 & 94.17 & 93.33 \\
\hline Average & $79.79 \pm 1.23$ & $94.79 \pm 1.23$ & $95.62 \pm 1.49$
\end{tabular}

\subsubsection{Results}

Tab. 5 presents the classification results for the recognition of drop caps images. We report the accuracy scores obtained for the different segmentation methods, as well as for GFDs and FHistograms. From these results, we can see that interlacement descriptors are able to efficiently classify the different types of background styles depicted in this dataset. They outperform GFD descriptors on all segmentation methods, and they compete with FHistograms, while capturing a different kind of spatial information. Indeed, FHistograms mainly characterize the overall mass repartition of the two objects, while our approach takes into account their directional interlacement patterns.

\subsection{Classification and Retrieval of Urban Objects}

\subsubsection{Dataset}

For this application, the dataset ${ }^{2}$ used is a very high spatial resolution (VHSR) image $\left(10^{8}\right.$ pixels) from the PLÉIADES satellite, representing the urban area of Strasbourg, France. This image was segmented using a multiresolution bottomup algorithm [41] dedicated to the processing of VHSR images. The parameters of this algorithm were chosen in order to obtain a fine image partition, where segments could match with elementary urban objects. These segments were then labeled using a supervised classification approach with four semantic classes: buildings, roads, vegetation and water. This dataset also comes with a reference map of the different urban blocks in the image, where each block is delineated and labeled with its corresponding class. In these experiments, we used two classes: continuous (highdensity blocks) and discontinuous (low-density blocks). This results in 617 images, each representing an urban block, with different sizes and shapes, and presenting different spatial configurations of urban objects. As illustrated in Fig. 14, such urban blocks appear as very spectrally and geometrically heterogeneous, since they model a complex spatial composition of basic urban objects [42].

\subsubsection{Experimental Protocol}

The first experiment is the classification of urban blocks as continuous or discontinuous. Indeed, independently from their shapes or sizes, such blocks are composed of distinctive spatial configurations of buildings and vegetation objects. For each urban block image, we compute the enlacement and interlacement descriptors between its buildings pixels

2. This work is partially supported by the COCLICO project, funded by the French Research Agency (ANR Modèles Numériques, ANR-12MN-001-COCLICO, 2012-2016). 


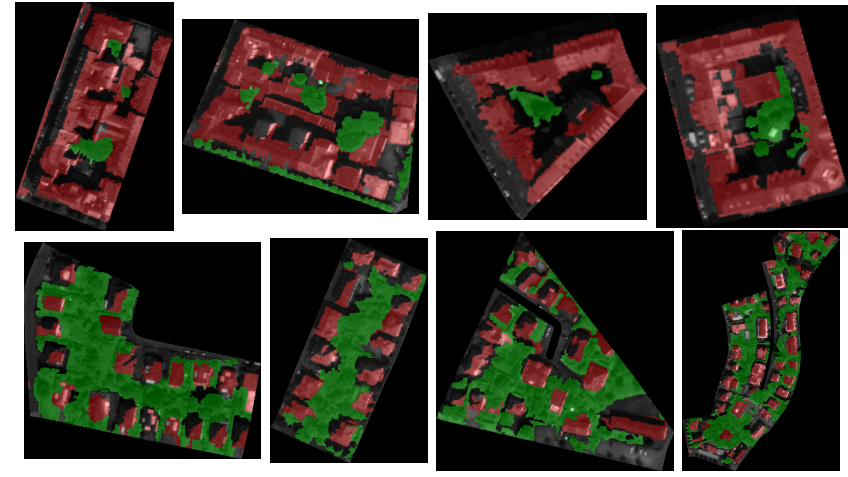

Fig. 14. Examples of urban blocks from the VHSR satellite image. First line: continuous urban blocks. Second line: discontinuous urban blocks. The overlays represent the objects in the blocks (red: buildings, green: vegetation), which were obtained with a segmentation method.

(object $A$ ) and vegetation ones (object $B$ ). As for the previous applications, we employ the same classification protocol: $k$-NN classifier, leave-one-out cross validation, and comparison with FHistograms and GFDs.

The second experiment is to perform semantic queries for the retrieval of urban scenes, based on enlacement or interlacement configurations between any combination of urban objects. A typical semantic query could be formulated in natural language, for instance: "retrieve images with the highest interlacement between buildings and roads". Here, we did not use urban blocks described previously, but an exhaustive split of the initial segmented image into 396 square blocks of $500 \times 500$ pixels. We then employed our averaging strategy described in Section 5.1 in order to obtain scalar evaluations from the descriptors (averaging in the $[0,2 \pi]$ interval). These values are then sorted, and images can be retrieved based on their overall quantity of enlacement or interlacement.

\subsubsection{Results}

Fig. 15 shows the precision-recall curves obtained for the classification of urban blocks as continuous or discontinuous. We report results for enlacement $\left(\mathcal{E}_{A B}\right.$ and $\left.\mathcal{E}_{B A}\right)$ and interlacement $\left(\mathcal{I}_{A B}\right)$ descriptors, as well as for GFDs and FHistograms. We observe that the best results are obtained for $\mathcal{E}_{A B}$, describing how buildings are enlaced by vegetation. This is a rather distinctive aspect between continuous and discontinuous urban blocks. On the contrary, interlacement descriptors $\left(\mathcal{I}_{A B}\right)$ perform rather poorly for this specific application. This might be due to the fact that many urban blocks do not present a mutual enlacement of objects, but instead a relative enlacement only. This reinforces our idea that enlacement and interlacement spatial relations can be used as different concepts, independently from each other, depending on the application requirements.

Fig. 16 shows the results of several semantic queries performed in the context of our second experiment. Each row presents retrieved images (top, intermediate and last results) of semantic queries for different pairs of objects. Note that we discard images where the requested objects are not present. The three first queries show interlacement configurations of objects. The fourth query is different, because it shows enlacement configurations of an object by

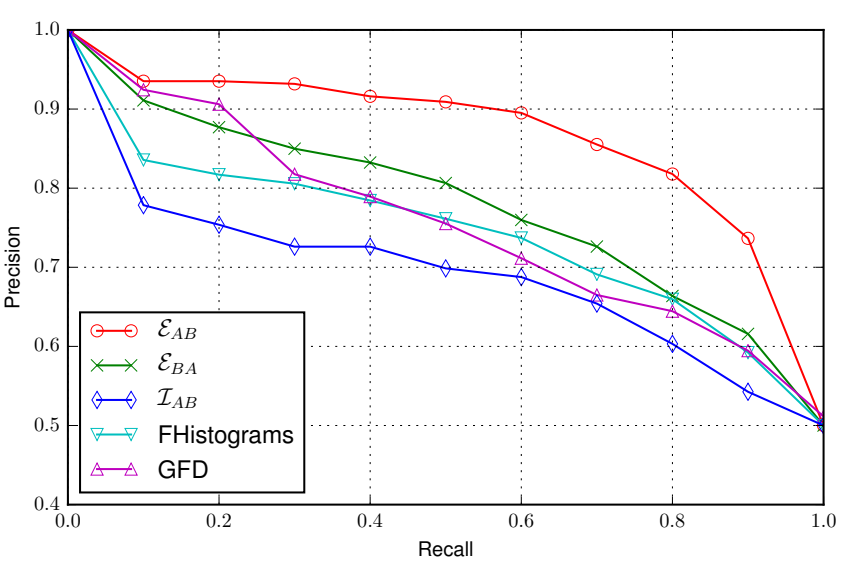

Fig. 15. Precision-recall curves obtained for the classification of urban blocks into continuous and discontinuous, for enlacement and interlacement descriptors, as well as for FHistograms and GFDs.

another. These retrieval results suggest that enlacement and interlacement descriptors can be used to perform semantic queries to efficiently retrieve areas with complex spatial organizations of geographical objects.

\subsection{Heatmaps}

Finally, we also propose a strategy to visualize more in details the results of our enlacement features, by building heatmaps modeling the individual contribution of each pixel in the computation of the enlacement values between two objects. When computing the enlacement $\mathcal{E}_{A B}$, for each pixel of $A$, we keep track of the number of times it is counted as an argument towards the enlacement of $A$ by $B$. Conversely, we do the same for each pixel of $B$ when computing $\mathcal{E}_{B A}$. A heatmap is then built by assigning to each pixel a color value reflecting its local enlacement degree.

To illustrate this approach, two heatmaps for retinal images are presented in Fig. 17. We can observe the stronger interlacement of blood vessels in the normal case, as opposed to the pathological case. This visualization approach can be useful in many practical cases.

\section{Conclusion}

In this article, we introduced an approach for the modeling of complex spatial configurations of objects represented in images. We defined the enlacement and interlacement spatial relations as a generalization of the notions of surrounding and imbrication of objects. Based on these definitions, we proposed new relative position descriptors, designed to characterize the enlacement and the interlacement of objects in a directional manner. These descriptors can handle the case of arbitrarily shaped objects, potentially composed of multiple connected components. We also put an emphasis on their invariance properties, which are often required in many pattern recognition tasks.

We presented experiments on different applications, illustrating the performance and the genericity of our approach. First, we showed how enlacement descriptors can be used in a fuzzy evaluation framework for the interpretation 


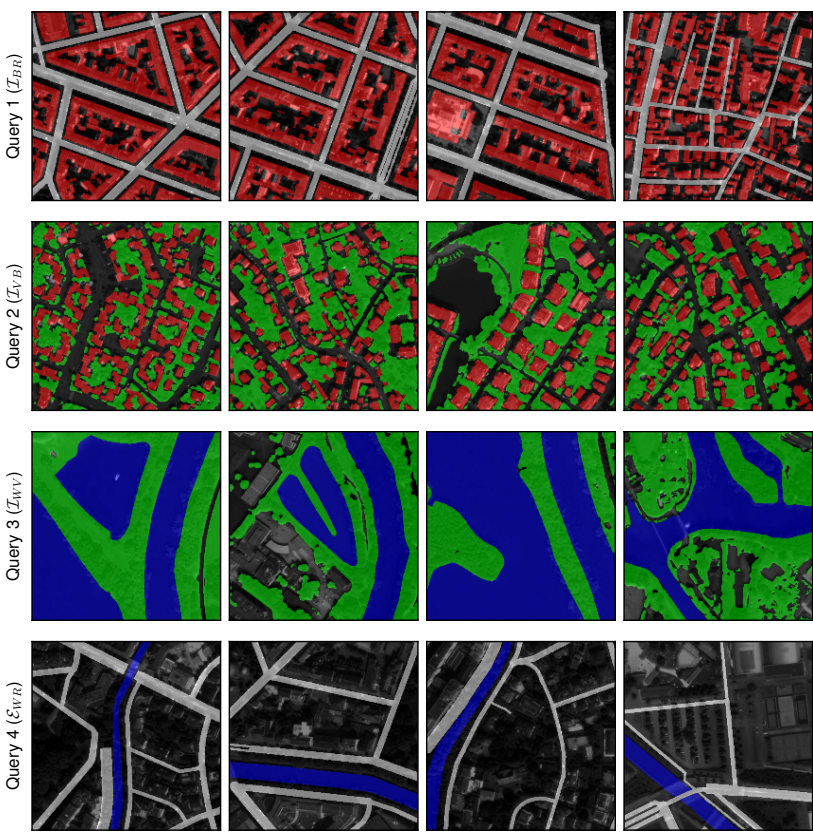

(a) Top retrieved results
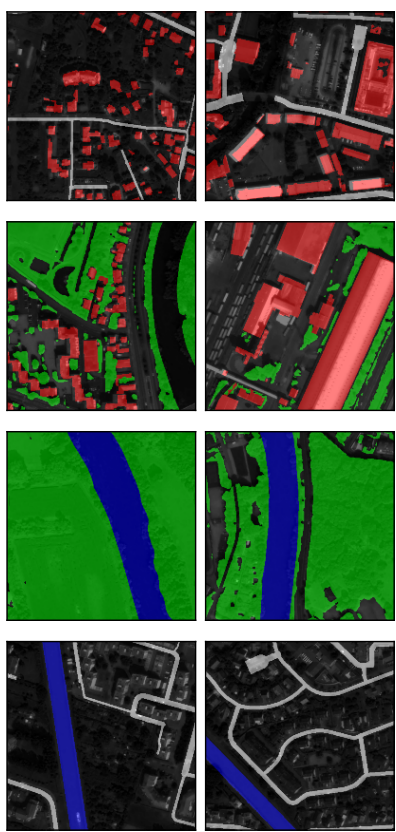

(b) Intermediate retrieved results
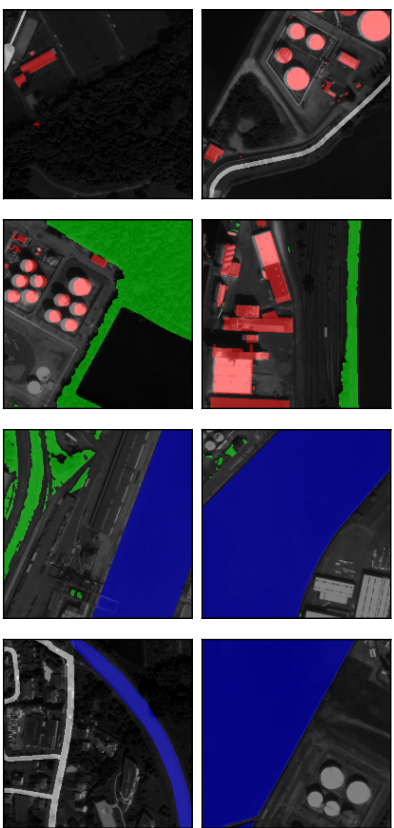

(c) Last retrieved results

Fig. 16. Semantic queries based on the enlacement or interlacement between different pairs of objects (each row is a different query). For each query, we show different levels of retrieved images: (a) the top four results, (b) two intermediate and (c) the two last images. First row: interlacement between buildings (red) and roads (gray). Second row: interlacement between buildings (red) and vegetation (green). Third row: interlacement between water (blue) and vegetation (green). Fourth row: enlacement of water (blue) by roads (gray).

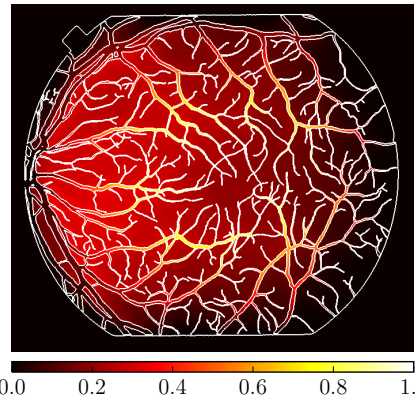

(a)

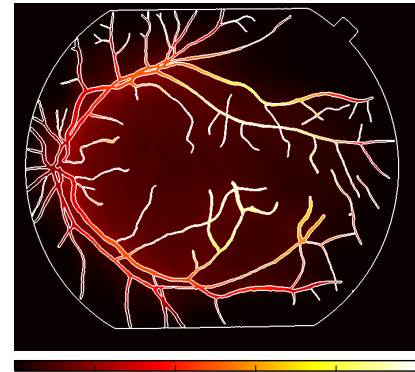

(b)
Fig. 17. Heatmaps showing the local interlacement between different objects. (a) Healthy fundus. (b) Pathological fundus (diabetic retinopathy).

of complex spatial relations. Then, we proposed quantitative validations in the application domains of medical imaging, document analysis, and remote sensing.

In future works, we plan to extend our approach by integrating a measure of spacing in interlacement configurations, allowing to better take into account the distance between the objects. We also plan to combine this approach with other types of relative position descriptors, allowing to build composite vocabularies of spatial relations. Finally, we would like to further explore how to integrate such descriptors into recognition frameworks based on local features.

\section{REFERENCES}

[1] T. P. McNamara, "Mental representations of spatial relations," Cognitive Psychology, vol. 18, no. 1, pp. 87-121, 1986.

[2] D. Marr, Vision: A Computational Investigation into the Human Representation and Processing of Visual Information. MIT Press, 1982.

[3] J. Freeman, "The modelling of spatial relations," Computer Graphics and Image Processing, vol. 4, no. 2, pp. 156-171, 1975.
[4] I. Bloch, "Fuzzy sets for image processing and understanding," Fuzzy Sets and Systems, vol. 281, pp. 280-291, 2015.

[5] I. Bloch and A. Ralescu, "Directional relative position between objects in image processing: A comparison between fuzzy approaches," Pattern Recognition, vol. 36, no. 7, pp. 1563-1582, 2003.

[6] I. Bloch, "Fuzzy spatial relationships for image processing and interpretation: A review," Image and Vision Computing, vol. 23, no. 2, pp. 89-110, 2005.

[7] I. Bloch, O. Colliot, and R. M. Cesar, "On the ternary spatial relation "between"," IEEE Transactions on Systems, Man, and Cybernetics, Part B: Cybernetics, vol. 36, no. 2, pp. 312-327, 2006.

[8] P. Matsakis, L. Wendling, and J. Ni, "A general approach to the fuzzy modeling of spatial relationships," in Methods for Handling Imperfect Spatial Information, 2010, pp. 49-74.

[9] I. Bloch, "Fuzzy relative position between objects in image processing: A morphological approach," IEEE Transactions on Pattern Analysis and Machine Intelligence, vol. 21, no. 7, pp. 657-664, 1999.

[10] R. M. Cesar, E. Bengoetxea, and I. Bloch, "Inexact graph matching using stochastic optimization techniques for facial feature recognition," in International Conference on Pattern Recognition (ICPR), vol. 2, 2002, pp. 465-468.

[11] O. Colliot, O. Camara, and I. Bloch, "Integration of fuzzy spatial relations in deformable models - Application to brain MRI segmentation," Pattern Recognition, vol. 39, no. 8, pp. 1401-1414, 2006.

[12] A. Delaye and E. Anquetil, "Learning of fuzzy spatial relations between handwritten patterns," International Journal on Data Mining, Modelling and Management, vol. 6, no. 2, pp. 127-147, 2014.

[13] K. Miyajima and A. Ralescu, "Spatial organization in 2D segmented images: Representation and recognition of primitive spatial relations," Fuzzy Sets and Systems, vol. 65, no. 2, pp. 225-236, 1994.

[14] P. Matsakis and L. Wendling, "A new way to represent the relative position between areal objects," IEEE Transactions on Pattern Analysis and Machine Intelligence, vol. 21, no. 7, pp. 634-643, 1999.

[15] P. Matsakis, J. M. Keller, O. Sjahputera, and J. Marjamaa, “The use of force histograms for affine-invariant relative position description," IEEE Transactions on Pattern Analysis and Machine Intelligence, vol. 26, no. 1, pp. 1-18, 2004.

[16] J. Ni and P. Matsakis, "An equivalent definition of the histogram of forces: Theoretical and algorithmic implications," Pattern Recognition, vol. 43, no. 4, pp. 1607-1617, 2010. 
[17] P. Matsakis, J. M. Keller, L. Wendling, J. Marjamaa, and O. Sjahputera, "Linguistic description of relative positions in images," IEEE Transactions on Systems, Man, and Cybernetics, Part B: Cybernetics, vol. 31, no. 4, pp. 573-88, 2001.

[18] A. R. Buck, J. M. Keller, and M. Skubic, "A memetic algorithm for matching spatial configurations with the histograms of forces," IEEE Transactions on Evolutionary Computation, vol. 17, no. 4, pp. 588-604, 2013.

[19] S. Tabbone and L. Wendling, "Color and grey level object retrieval using a 3D representation of force histogram," Image and Vision Computing, vol. 21, no. 6, pp. 483-495, 2003.

[20] M. Clément, M. Garnier, C. Kurtz, and L. Wendling, "Color object recognition based on spatial relations between image layers," in International Conference on Computer Vision Theory and Applications (VISAPP), 2015, pp. 427-434.

[21] P. Matsakis, M. Naeem, and F. Rahbarnia, "Introducing the $\Phi$ Descriptor - A Most Versatile Relative Position Descriptor," in International Conference on Pattern Recognition Applications and Methods (ICPRAM), 2015, pp. 87-98.

[22] J. F. Allen, "Maintaining knowledge about temporal intervals," Communications of the ACM, vol. 26, no. 11, pp. 832-843, 1983.

[23] M. Naeem and P. Matsakis, "Relative Position Descriptors - A Review," in International Conference on Pattern Recognition Applications and Methods (ICPRAM), 2015, pp. 286-295.

[24] B. Fan, F. Wu, and Z. Hu, "Rotationally invariant descriptors using intensity order pooling," IEEE Transactions on Pattern Analysis and Machine Intelligence, vol. 34, no. 10, pp. 2031-2045, 2012.

[25] Z. Wang, B. Fan, G. Wang, and F. Wu, "Exploring local and overall ordinal information for robust feature description," IEEE Transactions on Pattern Analysis and Machine Intelligence, vol. 38, no. 11, pp. 2198-2211, 2015.

[26] A. Rosenfeld and R. Klette, "Degree of adjacency or surroundedness," Pattern Recognition, vol. 18, no. 2, pp. 169-177, 1985.

[27] M. C. Vanegas, I. Bloch, and J. Inglada, "A fuzzy definition of the spatial relation "surround" - Application to complex shapes," in European Society for Fuzzy Logic and Technology (EUSFLAT), 2011, pp. 844-851.

[28] P. Matsakis and S. Andréfouët, "The fuzzy line between among and surround," in IEEE International Conference on Fuzzy Systems (FUZ-IEEE), vol. 2, 2002, pp. 1596-1601.

[29] N. Loménie and D. Racoceanu, "Point set morphological filtering and semantic spatial configuration modeling: Application to microscopic image and bio-structure analysis," Pattern Recognition, vol. 45, no. 8, pp. 2894-2911, 2012.

[30] C. M. Takemura, R. M. Cesar, and I. Bloch, "Modeling and measuring the spatial relation along: Regions, contours and fuzzy sets," Pattern Recognition, vol. 45, no. 2, pp. 757-766, 2012.

[31] M. C. Vanegas, I. Bloch, and J. Inglada, "Alignment and parallelism for the description of high-resolution remote sensing images," IEEE Transactions on Geoscience and Remote Sensing, vol. 51, no. 6, pp. 3542-3557, 2013.

[32] _ _ "Fuzzy spatial relations for high resolution remote sensing image analysis: The case of "to go across"," in IEEE Geoscience and Remote Sensing Symposium (IGARSS), vol. 4, 2009, pp. 773-776.

[33] D. Dubois, H. Prade, and C. Testemale, "Weighted fuzzy pattern matching," Fuzzy sets and systems, vol. 28, no. 3, pp. 313-331, 1988.

[34] D. Zhang and G. Lu, "Shape-based image retrieval using generic Fourier descriptor," Signal Processing: Image Communication, vol. 17, no. 10, pp. 825-848, 2002.

[35] J. J. Staal, M. D. Abramoff, M. Niemeijer, M. A. Viergever, and B. Van Ginneken, "Ridge based vessel segmentation in color images of the retina," IEEE Transactions on Medical Imaging, vol. 23, no. 4, pp. 501-509, 2004.

[36] M. M. Fraz, P. Remagnino, A. Hoppe, B. Uyyanonvara, A. R. Rudnicka, C. G. Owen, and S. A. Barman, "An ensemble classificationbased approach applied to retinal blood vessel segmentation," IEEE Transactions on Biomedical Engineering, vol. 59, no. 9, pp. 25382548, 2012.

[37] J. Orlando, E. Prokofyeva, and M. Blaschko, “A Discriminatively Trained Fully Connected Conditional Random Field Model for Blood Vessel Segmentation in Fundus Images," IEEE Transactions on Biomedical Engineering, vol. PP, 2016.

[38] J. Odstrcilik, R. Kolar, A. Budai, J. Hornegger, J. Jan, J. Gazarek, T. Kubena, P. Cernosek, O. Svoboda, and E. Angelopoulou, "Retinal vessel segmentation by improved matched filtering: Evaluation on a new high-resolution fundus image database," IET Image Processing, vol. 7, no. 4, pp. 373-383, 2013.
[39] M. D. Abràmoff, J. M. Reinhardt, S. R. Russell, J. C. Folk, V. B. Mahajan, M. Niemeijer, and G. Quellec, "Automated early detection of diabetic retinopathy," Ophthalmology, vol. 117, no. 6, pp. 1147-1154, 2010.

[40] M. Coustaty, R. Pareti, N. Vincent, and J. M. Ogier, "Towards historical document indexing: Extraction of drop cap letters," International Journal on Document Analysis and Recognition, vol. 14 no. 3, pp. 243-254, 2011.

[41] M. Baatz and A. Schape, "Multiresolution segmentation: An optimization approach for high quality multi-scale image segmentation," in Angewandte Geographische Informationsverarbeitung XII, 2000, pp. 12-23.

[42] C. Kurtz, N. Passat, P. Gançarski, and A. Puissant, "Extraction of complex patterns from multiresolution remote sensing images: A hierarchical top-down methodology," Pattern Recognition, vol. 45, no. 2, pp. 685-706, 2012.

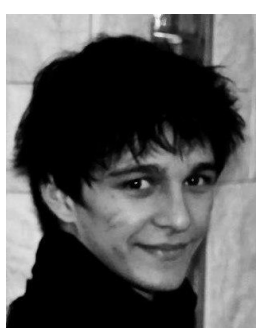

Michaël Clément received the Engineering degree from Grenoble INP - Ensimag in 2013 and the MSc degree in Computer Science from the Université Paris Descartes in 2014. He is currently a PhD student at the LIPADE lab, Paris. His research interests include computer vision, pattern recognition, content-based image retrieval and spatial relations.

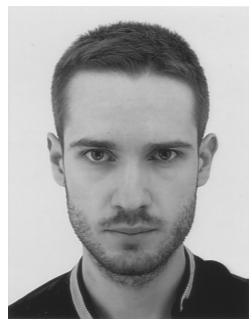

Adrien Poulenard received the BSc degree and the MSc degree in fundamental and applied mathematics from the Universite Paris-Sud, in 2013 and 2015. In 2014, he did a research internship in the SIP team of the LIPADE lab, Paris. His main research interests are algebraic geometry and artificial intelligence.

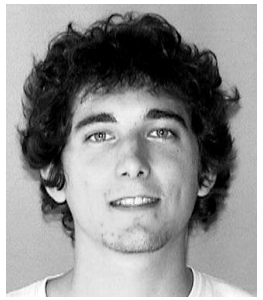

Camille Kurtz obtained the MSc and PhD in Computer Science from the Université de Strasbourg, France, in 2009 and 2012. He was a postdoctoral fellow at Stanford University, CA, USA between 2012 and 2013. He is now an associate professor at Université Paris Descartes, France and a member of the SIP team, LIPADE. His scientific interests include image analysis, data mining, medical imaging and remote sensing.

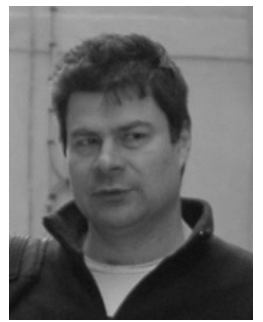

Laurent Wendling received the PhD degree in Computer Science in 1997 from the Université Paul Sabatier, Toulouse, France. He received the habilitation degree in 2006. From 1999 to 2009, he was an assistant professor at the ESIAL Nancy, and a member of LORIA. He is currently a full professor at the Université Paris Descartes. $\mathrm{He}$ is also a member of the SIP team, LIPADE. His current research topics are spatial relations, feature selection, and image segmentation. 


\section{APPENDIX}

Proof of Property 1. By definition, for any $\theta, t \in \mathbb{R}$,

$$
f_{A}^{(\theta+\pi, \rho)}(t)=f_{A}^{(\theta,-\rho)}(-t) .
$$

Using change of variables $(u, v, w, h)=-(x, y, z, \rho)$ in the expression of $E_{A B}(\theta+\pi)$ we obtain:

$$
\begin{aligned}
& E_{A B}(\theta+\pi) \\
& =\int_{\mathbb{R}^{2}} f_{B}^{(\theta, h)}(u) \int_{-\infty}^{u} f_{A}^{(\theta, h)}(v) \int_{-\infty}^{v} f_{B}^{(\theta, h)}(w) \mathrm{d} w \mathrm{~d} v \mathrm{~d} u \mathrm{~d} h \\
& =\int_{\mathbb{R}^{4}} f_{B}^{(\theta, h)}(u) f_{A}^{(\theta, h)}(v) f_{B}^{(\theta, h)}(w) \mathbb{1}_{\{u \geqslant v \geqslant w\}} \mathrm{d} w \mathrm{~d} v \mathrm{~d} u \mathrm{~d} h .
\end{aligned}
$$

Now using change of variable $(u, v, w)=(z, y, x)$ we have:

$$
\begin{aligned}
& E_{A B}(\theta+\pi) \\
& =\int_{\mathbb{R}^{4}} f_{B}^{(\theta, h)}(z) f_{A}^{(\theta, h)}(y) f_{B}^{(\theta, h)}(x) \mathbb{1}_{\{z \geqslant y \geqslant z\}} \mathrm{d} x \mathrm{~d} y \mathrm{~d} z \mathrm{~d} h \\
& =\int_{\mathbb{R}^{2}} f_{B}^{(\theta, h)}(x) \int_{x}^{+\infty} f_{A}^{(\theta, h)}(y) \int_{y}^{+\infty} f_{B}^{(\theta, h)}(z) \mathrm{d} z \mathrm{~d} y \mathrm{~d} x \mathrm{~d} h \\
& =E_{A B}(\theta) .
\end{aligned}
$$

Proof of Property 2. The translation $T_{v}$ is defined for any function $C: \mathbb{R}^{2} \rightarrow \mathbb{R}$ by:

$$
T_{v}(C)(x)=C(x-v) .
$$

Therefore we have for any $\theta, \rho, t \in \mathbb{R}$ :

$$
\begin{aligned}
f_{T_{v}(C)}^{(\theta, \rho)}(t) & =T_{v}(C)\left(e^{i \theta}(t+i \rho)\right) \\
& =C\left(e^{i \theta}(t+i \rho)-v\right) \\
& =C\left(e^{i \theta}\left(t+i \rho-e^{-i \theta} v\right)\right) \\
& =C\left(e^{i \theta}\left(t-\operatorname{Re}\left(e^{-i \theta} v\right)+i\left(\rho-\operatorname{Im}\left(e^{-i \theta} v\right)\right)\right)\right. \\
& =f_{C}^{\left(\theta, \rho-\operatorname{Im}\left(e^{-i \theta} v\right)\right)}\left(t-\operatorname{Re}\left(e^{-i \theta} v\right)\right)
\end{aligned}
$$

Hence, we have:

$$
\begin{aligned}
& \int_{\mathbb{R}} E\left(f_{T_{v}(A)}^{(\theta, \rho)}, f_{T_{v}(B)}^{(\theta, \rho)}\right) \mathrm{d} \rho \\
& =\int_{\mathbb{R}} E\left(f_{A}^{\left(\theta, \rho-\operatorname{Im}\left(e^{-i \theta} v\right)\right)}, f_{B}^{\left(\theta, \rho-\operatorname{Im}\left(e^{-i \theta} v\right)\right)}\right) \mathrm{d} \rho \\
& =\int_{\mathbb{R}} E\left(f_{A}^{(\theta, \rho)}, f_{B}^{(\theta, \rho)}\right) \mathrm{d} \rho .
\end{aligned}
$$

Proof of Property 3. By definition we have for any function $C: \mathbb{R}^{2} \rightarrow \mathbb{R}:$

$$
R_{\alpha}(C)=C\left(e^{-i \alpha} \bullet\right),
$$

hence for any $\theta, \rho \in \mathbb{R}$ we verify that:

$$
f_{R_{\alpha}(C)}^{(\theta, \rho)}=f_{C}^{(\theta-\alpha, \rho)}
$$

so that:

$$
\begin{aligned}
E_{R_{\alpha}(A) R_{\alpha}(B)}(\theta) & =\int_{\mathbb{R}} E\left(f_{R_{\alpha}(A)}^{(\theta, \rho)}, f_{R_{\alpha}(B)}^{(\theta, \rho)}\right) \mathrm{d} \rho \\
& =\int_{\mathbb{R}} E\left(f_{A}^{(\theta-\alpha, \rho)}, f_{B}^{(\theta-\alpha, \rho)}\right) \mathrm{d} \rho \\
& =E_{A B}(\theta-\alpha) .
\end{aligned}
$$

Proof of Property 4. Let $C: \mathbb{R}^{2} \rightarrow \mathbb{R}$, we have for any $\theta, \rho \in$ $\mathbb{R}$ and any $t \in \mathbb{R}$ :

$$
\begin{aligned}
f_{S_{\lambda}(C)}^{(\theta, \rho)}(t) & =S_{\lambda}(C)\left(e^{i \theta}(t+i \rho)\right) \\
& =C\left(e^{i \theta}\left(\frac{t}{\lambda}+i \frac{\rho}{\lambda}\right)\right) \\
& =f_{C}^{(\theta, \rho / \lambda)}\left(\frac{t}{\lambda}\right)
\end{aligned}
$$

Hence:

$$
E\left(f_{S_{\lambda}(A)}^{(\theta, \rho)}, f_{S_{\lambda}(B)}^{(\theta, \rho)}\right)=E\left(f_{A}^{(\theta, \rho / \lambda)}\left(\frac{\bullet}{\lambda}\right), f_{B}^{(\theta, \rho / \lambda)}\left(\frac{\bullet}{\lambda}\right)\right) .
$$

Let $f, g: \mathbb{R} \rightarrow \mathbb{R}$ be measurable bounded functions with compact support. By change of variable $w=\frac{z}{\lambda}, v=\frac{y}{\lambda}$ and $u=\frac{x}{\lambda}$, we have:

$$
\begin{aligned}
& E\left(f\left(\frac{\bullet}{\lambda}\right), g\left(\frac{\bullet}{\lambda}\right)\right) \\
& =\int_{\mathbb{R}} f\left(\frac{x}{\lambda}\right) \int_{x}^{+\infty} f\left(\frac{y}{\lambda}\right) \int_{y}^{+\infty} g\left(\frac{z}{\lambda}\right) \mathrm{d} z \mathrm{~d} y \mathrm{~d} x \\
& \underset{\substack{w=\frac{z}{\lambda} \\
=}}{ } \lambda \int_{\mathbb{R}} f\left(\frac{x}{\lambda}\right) \int_{x}^{+\infty} f\left(\frac{y}{\lambda}\right) \int_{\frac{y}{\lambda}}^{+\infty} g(w) \mathrm{d} w \mathrm{~d} y \mathrm{~d} x \\
& \underset{\substack{v=\frac{y}{\lambda} \\
=}}{2} \int_{\mathbb{R}} f\left(\frac{x}{\lambda}\right) \int_{\frac{x}{\lambda}}^{+\infty} f(v) \int_{y}^{+\infty} g(w) \mathrm{d} w \mathrm{~d} v \mathrm{~d} x \\
& \underset{\substack{u=\frac{x}{\lambda} \\
=}}{3} \int_{\mathbb{R}} f(u) \int_{\frac{x}{\lambda}}^{+\infty} f(v) \int_{y}^{+\infty} g(w) \mathrm{d} w \mathrm{~d} v \mathrm{~d} u \\
& =\lambda^{3} E(f, g) \text {. }
\end{aligned}
$$

By applying this to $f=f_{A}^{(\theta, \rho / \lambda)}$ and $g=f_{B}^{(\theta, \rho / \lambda)}$ we obtain:

$$
\begin{aligned}
E_{S_{\lambda}(A) S_{\lambda}(B)}(\theta) & =\int_{\mathbb{R}} E\left(f_{S_{\lambda}(A)}^{(\theta, \rho)}, f_{S_{\lambda}(B)}^{(\theta, \rho)}\right) \mathrm{d} \rho \\
& =\int_{\mathbb{R}} E\left(f_{A}^{(\theta, \rho / \lambda)}\left(\frac{\bullet}{\lambda}\right), f_{B}^{(\theta, \rho / \lambda)}\left(\frac{\bullet}{\lambda}\right)\right) \mathrm{d} \rho \\
& =\lambda^{3} \int_{\mathbb{R}} E\left(f_{A}^{(\theta, \rho / \lambda)}, f_{B}^{(\theta, \rho / \lambda)}\right) \mathrm{d} \rho \\
& =\lambda^{4} \int_{\mathbb{R}} E\left(f_{A}^{(\theta, h)}, f_{B}^{(\theta, h)}\right) \mathrm{d} h \\
h & =\frac{\rho}{\lambda} \\
& =\lambda^{4} E_{A B}(\theta) .
\end{aligned}
$$

Proof of Property 5. Let $\lambda \in \mathbb{R}$ be a scaling factor. By definition, for any object $A$, we have:

$$
\left\|S_{\lambda}(A)\right\|_{1}=\lambda^{2}\|A\|_{1} .
$$

Hence for any objects $A$ and $B$ :

$$
\begin{aligned}
\mathcal{E}_{S_{\lambda}(A) S_{\lambda}(B)}(\theta) & =\frac{E_{S_{\lambda}(A) S_{\lambda}(B)}(\theta)}{\left\|S_{\lambda}(A)\right\|_{1}\left\|S_{\lambda}(B)\right\|_{1}} \\
& =\frac{\lambda^{4} E_{A B}(\theta)}{\lambda^{4}\|A\|_{1}\|B\|_{1}} \\
& =\mathcal{E}_{A B}(\theta) .
\end{aligned}
$$

